\title{
Basic Methods for Computing Special Functions
}

\author{
Amparo Gil \\ Departamento de Matemática Aplicada y CC. de la Computación. \\ ETSI Caminos. Universidad de Cantabria. 39005-Santander, Spain. \\ Javier Segura \\ Departamento de Matemáticas, Estadística y Computación, \\ Univ. de Cantabria, 39005 Santander, Spain. \\ Nico M. Temme \\ CWI, Science Park 123, 1098 XG Amsterdam, The Netherlands. \\ e-mail: amparo.gil, javier.segura@unican.es, Nico.Temme@cwi.nl
}

September 26, 2009

\begin{abstract}
This paper gives an overview of methods for the numerical evaluation of special functions, that is, the functions that arise in many problems from mathematical physics, engineering, probability theory, and other applied sciences. We consider in detail a selection of basic methods which are frequently used in the numerical evaluation of special functions: converging and asymptotic series, including Chebyshev expansions, linear recurrence relations, and numerical quadrature. Several other methods are available and some of these will be discussed in less detail. We give examples of recent software for special functions where these methods are used. We mention a list of new publications on computational aspects of special functions available on our website.
\end{abstract}

2000 Mathematics Subject Classification: 65D20, 41A60, 33C05, 33C10, 33C15.

Keywords \& Phrases: numerical evaluation of special functions, Chebyshev expansions, quadrature methods, transformation of series, continued fractions, asymptotic analysis. 


\section{Contents}

1 Introduction 3

2 Convergent and divergent series $\quad 5$

2.1 Divergent expansions . . . . . . . . . . . . . . . 7

3 Linear recurrence relations $\quad 8$

3.1 A simple recurrence relation . . . . . . . . . . . . . . 9

3.2 Some elements of the general theory . . . . . . . . . . 10

3.3 Miller's algorithm . . . . . . . . . . . . . . . . . 11

3.4 Examples of hypergeometric functions and recursions . . . . . 13

3.4.1 Bessel functions . . . . . . . . . . . . . . . . . . 13

3.4.2 Kummer functions . . . . . . . . . . . . . . . . . . 14

3.4.3 Gauss hypergeometric functions . . . . . . . . . . . 15

3.4 .4 Legendre functions . . . . . . . . . . . . . . 15

3.4.5 Coulomb wave functions . . . . . . . . . . . . 15

3.4.6 Parabolic cylinder functions . . . . . . . . . . 16

4 Chebyshev expansions $\quad 16$

4.1 Clenshaw's summation method . . . . . . . . . . . 17

4.2 Methods for obtaining the coefficients . . . . . . . . 17

4.2.1 Tabled coefficients . . . . . . . . . . . 17

4.2 .2 Discretizing the integral . . . . . . . . . . . . 17

4.2 .3 Clenshaw's method . . . . . . . . . . . . . . 18

4.2.4 Known coefficients in terms of special functions . . . . 20

5 Quadrature methods $\quad 22$

5.1 The trapezoidal rule . . . . . . . . . . . . . . . 23

5.1 .1 The trapezoidal rule on $\mathbb{R} \ldots \ldots \ldots 26$

5.2 Complex contours . . . . . . . . . . . . . 27

6 The Stokes phenomenon $\quad 29$

6.1 The Airy function . . . . . . . . . . . . . . 29

6.2 The recent interest in the Stokes phenomenon . . . . . . . . 31

6.3 Exponentially small terms in the Airy expansions . . . . . . . 31

7 A selection of other methods $\quad 32$

7.1 Continued fractions . . . . . . . . . . . . . . 32

7.1.1 Incomplete gamma functions . . . . . . . . . . . 33

7.1 .2 Incomplete beta function . . . . . . . . . . . 34 
7.2 Sequence transformations . . . . . . . . . . . . . 35

7.2.1 Padé approximations . . . . . . . . . . . . 36

7.2.2 How to compute the Padé approximants . . . . . . . . 37

7.2.3 Nonlinear sequence transformations . . . . . . . . . 38

7.2 .4 Numerical examples . . . . . . . . . . . . . . . . 40

7.3 Other quadrature methods . . . . . . . . . . . . . . . . . 42

7.4 Numerical inversion of Laplace transforms . . . . . . . . . . 42

7.5 Computing zeros of special functions . . . . . . . . . 45

7.6 Uniform asymptotic expansions . . . . . . . . . . . . 47

7.7 Taylor expansion methods for ordinary differential equations . 49

7.8 Computing symmetric elliptic integrals . . . . . . . . . 50

7.9 Best rational approximations . . . . . . . . . . 51

8 Recent software and publications on methods for computing special functions $\quad \mathbf{5 2}$

8.1 A selection of recent software for special functions . . . . . 52

8.2 Recent literature on the computation of special functions . . 54

\section{Introduction}

For workers in the applied sciences the Handbook of Mathematical Functions with Formulas, Graphs, and Mathematical Tables [1], edited by Milton Abramowitz and Irene Stegun, and published in 1964 is usually the first source of information about the properties of special functions. It may be the most cited book in mathematics. These days the Handbook is being updated as a Digital Library of Mathematical Functions (DLMF), and will be freely accessible in a Web version. Other sources for collections of formulas for special functions on the web are Wolfram MathWorld ${ }^{1}$ and Wikipedia ${ }^{2}$.

These sources give many properties of special functions of which a number can be used for their numerical evaluation, sometimes with references to suitable algorithms. However, it is not always clear how to write efficient and reliable algorithms.

The present paper gives an overview on numerical methods for special functions. It is based on our recent book [55] in which we consider four Basic Methods, namely

1. Convergent and divergent series.

\footnotetext{
${ }^{1}$ http://mathworld.wolfram.com/

${ }^{2}$ http://en.wikipedia.org/
} 
2. Chebyshev expansions.

3. Linear recurrence relations and associated continued fractions.

4. Quadrature methods.

In addition we give a selection of published algorithms for special functions.

There are many other methods, which are also discussed in our book, and some of them will be discussed in this overview as well. For example, the use of differential equations will be discussed in connection with the Taylor expansion method for initial boundary problems.

Our general point of view in connection with special functions is to remain modest in the number of parameters. It is possible to design straightforward algorithms for the generalized hypergeometric function

$$
{ }_{p} F_{q}\left(\begin{array}{l}
a_{1}, \cdots, a_{p} \\
b_{1}, \cdots, b_{q}
\end{array} ; z\right)=\sum_{n=0}^{\infty} \frac{\left(a_{1}\right)_{n} \cdots\left(a_{p}\right)_{n}}{\left(b_{1}\right)_{n} \cdots\left(b_{q}\right)_{n}} \frac{z^{n}}{n !},
$$

where $p \leq q+1$ and $(a)_{n}$ is the Pochhammer symbol, also called the shifted factorial, defined by

$$
(a)_{0}=1, \quad(a)_{n}=a(a+1) \cdots(a+n-1)(n \geq 1), \quad(a)_{n}=\frac{\Gamma(a+n)}{\Gamma(a)} .
$$

Many special functions can be written in terms of this function, with the main cases given by $p=1, q=1$ (Kummer functions, with convergence of the series for all complex $z$ ) and $p=2, q=1$ (Gauss hypergeometric functions, with convergence if $|z|<1$ ). For efficient and reliable algorithms the series in (1.1) is only of limited use.

Also, differential equations, especially those that arise in the physical sciences, is the reservoir that generates many special functions. One may define a general second order equation, make an algorithm for this equation, and expect that solutions of simpler equations follow from the general solution. However, very difficult problems may arise then. Consider as an example the equation

$$
\frac{d^{2}}{d z^{2}} w(z)=\left(p z^{2}+q z+r\right) w(z)
$$

the solutions of which can be expressed in terms of the parabolic cylinder functions $U$ and $V$, see [1, Ch. 19], which are solutions of the equation

$$
\frac{d^{2}}{d z^{2}} w(z)=\left(\frac{1}{4} z^{2}+a\right) w(z)
$$


When $p=r=0$ in (1.3) that equation reduces to the Airy equation, whereas the Airy functions are no special cases of the parabolic cylinder functions $U$ and $V$ (in the sense as Airy functions are Bessel functions for certain values of the order of these functions). A nontrivial limiting process with $p \rightarrow 0$ and $r \rightarrow 0$ is needed to get Airy functions from a linear combination of the solutions of (1.3).

In its turn, the parabolic cylinder function $U(a, z)$ is a special case of the confluent hypergeometric function (also called Kummer functions) $U(a, c, z)$. We have two forms [1, p. 691]:

$$
\begin{aligned}
U(a, z) & =2^{-\frac{1}{4}-\frac{1}{2} a} e^{-\frac{1}{4} z^{2}} U\left(\frac{1}{2} a+\frac{1}{4}, \frac{1}{2}, \frac{1}{2} z^{2}\right) \\
& =2^{-\frac{3}{4}-\frac{1}{2} a} z e^{-\frac{1}{4} z^{2}} U\left(\frac{1}{2} a+\frac{3}{4}, \frac{3}{2}, \frac{1}{2} z^{2}\right) .
\end{aligned}
$$

The first form suggests that the function $U(a, z)$ is an even function of $z$, the second one that it is odd. The point is that this Kummer function is multi-valued, and the representation

$$
\begin{aligned}
U(a, \pm z)= & \frac{\sqrt{\pi} 2^{-\frac{1}{4}-\frac{1}{2} a} e^{-\frac{1}{4} z^{2}}}{\Gamma\left(\frac{3}{4}+\frac{1}{2} a\right)}{ }_{1} F_{1}\left(\begin{array}{c}
\frac{1}{2} a+\frac{1}{4} \\
\frac{1}{2} z^{2}
\end{array}\right) \mp \\
& \frac{\sqrt{\pi} 2^{\frac{1}{4}-\frac{1}{2} a} z e^{-\frac{1}{4} z^{2}}}{\Gamma\left(\frac{1}{4}+\frac{1}{2} a\right)}{ }_{1} F_{1}\left(\begin{array}{c}
\frac{1}{2} a+\frac{3}{4} ; \frac{1}{2} z^{2} \\
\frac{3}{2}
\end{array}\right)
\end{aligned}
$$

gives a better insight. However, this form is extremely unstable for intermediate or large values of $z$.

In our opinion it is important to have codes that can be used for a limited class of functions. In this sense we have written algorithms for conical functions $P_{-1 / 2+i \tau}^{\mu}(x)$ [56] for real $x, \tau$ and $\mu$, and not for Legendre functions of general complex degree. Also, we have written codes [46] for modified Bessel functions of purely imaginary order, that is for $K_{i a}(x)$ and a related function, and not a general code for Bessel functions with general complex order.

\section{Convergent and divergent series}

Convergent series for special functions usually arise in the form of hypergeometric series, with as general form the one shown in (1.1). The series is easy to evaluate because of the recursion $(a)_{n+1}=(a+n)(a)_{n}, n \geq 0$, of the Pochhammer symbols in (1.2). For certain special function, for example for 
the modified Bessel function

$$
I_{\nu}(z)=\left(\frac{1}{2} z\right)^{\nu} \sum_{n=0}^{\infty} \frac{\left(\frac{1}{4} z^{2}\right)^{n}}{\Gamma(\nu+n+1) n !}=\left(\frac{1}{2} z\right)_{0}^{\nu} F_{1}\left(\begin{array}{c}
- \\
\nu+1
\end{array} ; \frac{1}{4} z^{2}\right)
$$

it gives a stable representation when $z>0$ and $\nu \geq 0$ and it is an efficient representation when $z$ is not large compared with $\nu$. However, when we use this expansion in the representation of the other modified Bessel function

$$
K_{\nu}(z)=\frac{1}{2} \pi \frac{I_{-\nu}(z)-I_{\nu}(z)}{\sin \pi \nu},
$$

it can be used only for small values of $z$. This is because of the cancellation of numerical digits, which can be seen from the asymptotic estimates

$$
I_{\nu}(z) \sim \frac{e^{z}}{\sqrt{2 \pi z}}, \quad K_{\nu}(z) \sim \sqrt{\frac{\pi}{2 z}} e^{-z}, \quad z \rightarrow \infty,
$$

which is valid for fixed values of $\nu$.

There is another phenomenon when using combinations of hypergeometric functions. When $\nu$ is and integer, the form in (2.2) is well defined by a limiting process, but for numerical computations a special algorithm is needed. See [103], where it is shown that it is sufficient to treat the case $\nu \sim 0$ in detail and that the remaining integer values follow from recursion relations.

The case for confluent hypergeometric functions is more complicated. We have for the function $U(a, c, z)$ the representation

$$
U(a, c, z)=\frac{\pi}{\sin \pi c}\left(\frac{{ }_{1} F_{1}\left(\begin{array}{l}
a \\
c
\end{array} ; z\right)}{\Gamma(1+a-c) \Gamma(c)}-z^{1-c} \frac{{ }_{1} F_{1}\left(\begin{array}{c}
1+a-c \\
2-c
\end{array} ; z\right)}{\Gamma(a) \Gamma(2-c)}\right),
$$

and it is useful for small values of $z$. Consider $c \sim 0$. We have

$$
\lim _{c \rightarrow 0} \frac{{ }_{1} F_{1}\left(\begin{array}{l}
a \\
c
\end{array} ; z\right)}{\Gamma(1+a-c) \Gamma(c)}=\frac{z_{1} F_{1}\left(\begin{array}{c}
a+1 \\
2
\end{array} ; z\right)}{\Gamma(a)} .
$$

So, apart from this limit, another (simultaneous) limiting process for $c \rightarrow 0$ needs to be controlled, and also the extra parameter $a$ makes it more difficult to write a stable algorithm. A published algorithm seems not to be available for this case. 
For Gauss hypergeometric functions similar problems arise in the connection formulas, say the one writing a function with argument $z$ as a linear combination of two functions with argument $1-z$. See [33] for numerical algorithms.

Other instabilities occur when the parameters of the hypergeometric function become large and/or complex.

For Gauss and Kummer hypergeometric functions many other convergent expansions are available, for example in terms of Chebyshev polynomials and of Bessel functions; see [72, $\S \S 9.3 .4,9.4 .1,9.4 .3$,$] . For a different$ type of expansions in terms of Bessel functions, with an application to the parabolic cylinder functions, see [75].

\section{$2.1 \quad$ Divergent expansions}

With this we mean asymptotic expansions of the form

$$
F(z) \sim \sum_{n=0}^{\infty} \frac{c_{n}}{z^{n}}, \quad z \rightarrow \infty .
$$

The series usually diverges, but it has the property

$$
F(z)=\sum_{n=0}^{N-1} \frac{c_{n}}{z^{n}}+R_{N}(z), \quad R_{N}(z)=\mathcal{O}\left(z^{-N}\right), \quad z \rightarrow \infty,
$$

for $N=0,1,2, \ldots$, and the order estimate holds for fixed $N$. This is the Poincaré-type expansion and for special functions like the gamma and Bessel functions they are crucial for evaluating these functions. Other variants of the expansion are also important, in particular expansions that hold for a certain range of additional parameters (this leads to the uniform asymptotic expansions in terms of other special functions like Airy functions, which are useful in turning point problems).

Usually the optimal choice of $N$ with a given (large) $z$ occurs for the $N$ that makes $c_{N} / z^{N}$ the smallest term. And usually the error estimate in (2.7) may be exponentially small for this $N$. Say, with $z>0$, the smallest term is achieved when $N \sim z$, then it may happen that $R_{N}(N)=\mathcal{O}(\exp (-N))$. Many asymptotic expansions for special functions share this property, and it makes asymptotic expansions very useful for numerical computations.

Convergent power series may be very unstable in certain parts of their convergence region, as the expansion of $e^{-z}$ for $\Re z>0$. In a similar way, asymptotic expansions may be very useful in a certain sector of the complex 
$z$-plane, but may become useless in other sectors. Other expansions may be available in these sectors.

For example, consider the compound expansion of the Kummer function

$$
\begin{aligned}
\frac{1}{\Gamma(c)}{ }_{1} F_{1}\left(\begin{array}{l}
a \\
c
\end{array} ; z\right)= & \frac{e^{z} z^{a-c}}{\Gamma(a)}\left[\sum_{n=0}^{R-1} \frac{(c-a)_{n}(1-a)_{n}}{n ! z^{n}}+\mathcal{O}\left(|z|^{-R}\right)\right]+ \\
& \frac{z^{-a} e^{ \pm i \pi a}}{\Gamma(c-a)}\left[\sum_{n=0}^{S-1} \frac{(a)_{n}(1+a-c)_{n}}{n !(-z)^{n}}+\mathcal{O}\left(|z|^{-S}\right)\right],
\end{aligned}
$$

the upper sign being taken if $-\frac{1}{2} \pi<\operatorname{ph} z<\frac{3}{2} \pi$, the lower sign if $-\frac{3}{2} \pi<$ $\operatorname{ph} z<\frac{1}{2} \pi$. When $\Re z>0$ the second term can be neglected because of $e^{z}$ in front of the first term. We see that within a sector properly inside the sector $-\frac{1}{2} \pi<\operatorname{ph} z<\frac{1}{2} \pi$ we can work with one expansion, and in a sector containing the negative $z$-axis with another one. In sectors containing the imaginary axis we need both expansions.

The fact that an entire function, as this Kummer function, does not have a unique asymptotic expansion valid for all phases of $z$ will be explained in $\S 6$, where we discuss elements of the Stokes phenomenon.

A remarkable point is in this example that we have, say for $-\frac{1}{2} \pi+\delta<$ $\operatorname{ph} z<\frac{1}{2} \pi-\delta(\delta$ a small positive number), not only one expansion, but also an expansion that gives an exponentially small correction to the main expansion. For computations (and also for applications in physics) this may give interesting information. The role of exponentially small terms in asymptotics has been discussed in great detail the last twenty years. For many aspects from a physicists' point of view, we refer to The Devil's Invention: Asymptotic, Superasymptotic and Hyperasymptotic Series [12] for a lively introduction to this topic. ${ }^{3}$ In $\S 6$ we also discuss aspects of the role of exponentially small terms.

\section{$3 \quad$ Linear recurrence relations}

Many special functions of mathematical physics satisfy a three-term recurrence relations. We first give a simple relation and discuss stability and direction of recursion, which elements are important in the general theory.

\footnotetext{
${ }^{3}$ The "Devil's invention" refers to a quote from Niels Hendrik Abel (1828), who claimed "Divergent series are the invention of the devil, and it is shameful to base on them any demonstration whatsoever."
} 


\subsection{A simple recurrence relation}

The recurrence relations

$$
f_{n}=f_{n-1}-\frac{x^{n}}{n !}, \quad g_{n}=g_{n-1}+\frac{x^{n}}{n !}, \quad n=1,2, \ldots,
$$

with initial values $f_{0}=e^{x}-1, g_{0}=1$ have solutions

$$
f_{n}=\sum_{m=n+1}^{\infty} \frac{x^{m}}{m !}, \quad g_{n}=e^{x}-f_{n}=\sum_{m=0}^{n} \frac{x^{m}}{m !},
$$

which are in fact special cases of the incomplete gamma functions:

$$
f_{n}=e^{x} \frac{\gamma(n+1, x)}{n !}, \quad g_{n}=e^{x} \frac{\Gamma(n+1, x)}{n !}, \quad n=0,1,2, \ldots,
$$

Assume that $x>0$. Then, following our intuition, the recursion for $g_{n}$ will not cause any problem, since two positive numbers are always added during the recursion. For the recurrence relation of $f_{n}$ it is not clear, but there is a potential danger owing to the subtraction of two positive quantities. Note that the computation of the initial value $f_{0}$, for small values of $x$, may produce a large relative error, when the quantities $e^{x}$ and 1 are simply subtracted. This problem repeats itself for each subsequent $f_{n}$ that is computed by using the recurrence relation: in each step the next term of the Taylor series is subtracted from the exponential function.

Apparently, this is a hopeless procedure for computing successive $f_{n}$ (even when $x$ is not small). On the other hand, the computation of successive $g_{n}$ does not show any problem.

In the study of recurrence relations it may make sense to change the direction of the recursion. Writing the recursion for $f_{n}$ and $g_{n}$ in the backward direction:

$$
f_{n-1}=f_{n}+\frac{x^{n}}{n !}, \quad g_{n-1}=g_{n}-\frac{x^{n}}{n !}
$$

then we note that for both solutions the roles are reversed: $g_{n}$ is obtained by subtraction, whereas $f_{n}$ is obtained by addition of positive numbers. In addition, $\lim _{n \rightarrow \infty} f_{n}=0$.

It can be easily verified that both $f_{n}$ and $g_{n}$ satisfy the recurrence relation

$$
(n+1) y_{n+1}-(x+n+1) y_{n}+x y_{n-1}=0 .
$$

Again, this relation is stable for the computation of $g_{n}$ in the forward direction; it is stable for $f_{n}$ in the backward direction. Note that the solutions of 
this recursion satisfy $f_{n} \rightarrow 0, g_{n} \rightarrow e^{x}$ as $n \rightarrow \infty$. Apparently, the solution which becomes ultimately small in the forward direction (small compared to the other solution), is the victim. A similar phenomenon occurs in the backward direction. This phenomenon will be explained and put in a general framework in the following section.

\subsection{Some elements of the general theory}

For details on the theory of this topic we refer to [55, Chap. 4].

Consider the recurrence relation

$$
y_{n+1}+b_{n} y_{n}+a_{n} y_{n-1}=0, \quad n=1,2,3, \ldots,
$$

where $a_{n}$ and $b_{n}$ are given, with $a_{n} \neq 0$. Equation (3.6) is also called a linear homogeneous difference equation of the second order. In analogy with the theory of differential equations, two linearly independent solutions $f_{n}, g_{n}$ exist in general, with the property that any solution $y_{n}$ of (3.6) can be written in the form

$$
y_{n}=A f_{n}+B g_{n}
$$

where $A$ and $B$ do not depend on $n$. We are interested in the special case that the pair $\left\{f_{n}, g_{n}\right\}$ satisfies

$$
\lim _{n \rightarrow \infty} \frac{f_{n}}{g_{n}}=0 .
$$

Then, for any solution (3.7) with $B \neq 0$, we have $f_{n} / y_{n} \rightarrow 0$ as $n \rightarrow \infty$. When $B=0$ in (3.7), we call $y_{n}$ a minimal solution; when $B \neq 0$, we call $y_{n}$ a dominant solution. When we have two initial values $y_{0}, y_{1}$, assuming that $f_{0}, f_{1}, g_{0}, g_{1}$ are known as well, then we can compute $A$ and $B$. That is,

$$
A=\frac{g_{1} y_{0}-g_{0} y_{1}}{f_{0} g_{1}-f_{1} g_{0}}, \quad B=\frac{y_{0} f_{1}-y_{1} f_{0}}{g_{0} f_{1}-g_{1} f_{0}} .
$$

The denominators are different from 0 when the solutions $f_{n}, g_{n}$ are linearly independent.

When we assume that the initial values $y_{0}, y_{1}$ are to be used for generating a dominant solution, then $A$ may, or may not, vanish; $B$ should not vanish: $y_{0} f_{1} \neq y_{1} f_{0}$. When however the initial values are to be used for the computation of a minimal solution, then the much stronger condition $y_{0} f_{1}=y_{1} f_{0}$ should hold. It follows that, in this case, one and only one initial value can be prescribed and the other one follows from the relation $y_{0} f_{1}=y_{1} f_{0}$; in other words, the minimal solutions, if it exists, is unique 
up to a constant multiplicative factor. In the numerical approach this leads to the well-known instability phenomena for the computation of minimal solutions. The fact is that, when our initial values $y_{0}, y_{1}$ are not specified to an infinite precision, - and consequently $B$ does not vanish exactly - the computed solution (3.7) always contains a fraction of a dominant solution $g_{n}$. Hence, in the long run, our solution $y_{n}$ does not behave as a minimal solution, although we assumed that we were computing a minimal solution. This happens even if all further computations are done exactly.

In applications it is important to know whether a given equation (3.6) has dominant and minimal solutions. Often this can be easily concluded from the asymptotic behavior of the coefficients $a_{n}$ and $b_{n}$.

Assume that for large values of $n$ the coefficients $a_{n}, b_{n}$ behave as follows:

$$
a_{n} \sim a n^{\alpha}, \quad b_{n} \sim b n^{\beta}, \quad a b \neq 0
$$

with $\alpha$ and $\beta$ real; assume that $t_{1}, t_{2}$ are the zeros of the characteristic polynomial $\Phi(t)=t^{2}+b t+a$ with $\left|t_{1}\right| \geq\left|t_{2}\right|$. Then it follows from Perron's theorem [55, p. 93] that we have the following results.

1. If $\beta>\frac{1}{2} \alpha$ then the difference equation (3.6) has two linearly independent solutions $y_{n, 1}$ and $y_{n, 2}$, with the property

$$
\frac{y_{n+1,1}}{y_{n, 1}} \sim-b n^{\beta}, \quad \frac{y_{n+1,2}}{y_{n, 2}} \sim-\frac{a}{b} n^{\alpha-\beta}, \quad n \rightarrow \infty .
$$

2. If $\beta=\frac{1}{2} \alpha$ and $\left|t_{1}\right|>\left|t_{2}\right|$, then the difference equation (3.6) has two linear independent solutions $y_{n, 1}$ and $y_{n, 2}$, with the property

$$
\frac{y_{n+1,1}}{y_{n, 1}} \sim t_{1} n^{\beta}, \quad \frac{y_{n+1,2}}{y_{n, 2}} \sim t_{2} n^{\beta}, \quad n \rightarrow \infty,
$$

3. If $\beta=\frac{1}{2} \alpha$ and $\left|t_{1}\right|=\left|t_{2}\right|$, or if $\beta<\frac{1}{2} \alpha$, then some information is still available, but the theorem is inconclusive with respect to the existence of minimal and dominant solutions.

\subsection{Miller's algorithm}

This algorithm can be used for calculating a sequence

$$
f_{0}, f_{1}, \ldots, f_{N}
$$

of values of a minimal solution that satisfies (3.6); $N$ is a non-negative integer. Such sequences frequently occur in expansions of special functions; see for example the expansions in terms of Chebyshev polynomials in (4.16). 
When we use (3.6) in the backward direction we may start with two initial values $f_{N}$ and $f_{N-1}$. But these are perhaps difficult to obtain. Miller's algorithm does not need these values, and uses a smart idea for the computation of the required sequence (3.13). The algorithm works for many interesting cases and gives an efficient method for computing such sequences.

Assume we have a relation of the form

$$
S=\sum_{n=0}^{\infty} \lambda_{n} f_{n}, \quad S \neq 0 .
$$

The series should be convergent and $\lambda_{n}$ and $S$ should be known. The series in (3.14) plays a role in normalizing the required minimal solution. The series may be finite; we only require that at least one coefficient $\lambda_{n}$ with $n \leq N$ is different from zero. When just one coefficient, say $\lambda_{n}$, is different from zero, we assume that the value $f_{n}$ is available.

In Miller's algorithm a starting value $\nu$ is chosen, $\nu>N$, and a solution $\left\{y_{n}^{(\nu)}\right\}$ of (3.6) is computed with the false initial values

$$
y_{\nu+1}^{(\nu)}=0, \quad y_{\nu}^{(\nu)}=1 .
$$

The right-hand sides may be replaced by other values; at least one value should be different from zero. In some cases a judicious choice of these values may improve the convergence of the algorithm.

The computed solution $y_{n}$, with (3.15) as initial values, is a linear combination of the solutions $f_{n}$ and $g_{n}, g_{n}$ being a dominant solution. When we choose $\nu$ large enough it follows that the wanted solution $f_{n}$ satisfies $f_{n} \doteq \rho y_{n}, n=0,1, \ldots, N$, because the dominant solution $g_{n}$ can be neglected in the backward direction. For details and proofs we refer to [34] and $[55, \S 4.6]$. The number $\rho$ then follows from the normalizing sum (3.14). That is,

$$
\rho \doteq \frac{1}{S} \sum_{n=0}^{\nu} \lambda_{n} y_{n} .
$$

In [10] the above method was introduced for computing the modified Bessel functions $I_{n}(x)$. The recurrence relation for these functions reads

$$
I_{n+1}(x)+\frac{2 n}{x} I_{n}(x)-I_{n-1}(x)=0 .
$$

A normalizing condition (3.14) is

$$
e^{x}=I_{0}(x)+2 I_{1}(x)+2 I_{2}(x)+2 I_{3}(x) \cdots .
$$


Table 1: Computing the modified Bessel functions $I_{n}(x)$ for $x=1$ by using (3.17) in the backward direction. The underlined digits in the third column are correct.

\begin{tabular}{|c|c|c|}
\hline$n$ & $y_{n}$ before normalization & $y_{n} \doteq I_{n}(1)$ after normalization \\
\hline 0 & $2.28794930010^{+8}$ & $1.2660658780110^{-0}$ \\
\hline 1 & $1.021317610_{10^{+8}}^{+8}$ & $\overline{5.65159104106} 6_{10}^{-1}$ \\
\hline 2 & $2.453140800_{10^{+7}}$ & $1.3574766979410^{-1}$ \\
\hline 3 & $4.006129000_{10^{+6}}$ & $2.2168424928810^{-2}$ \\
\hline 4 & $4.94340000010^{+5}$ & $2.7371202216010^{-3}$ \\
\hline 5 & $4.905700000_{10}^{+4}$ & $2.7146315601210^{-4}$ \\
\hline 6 & $4.064000000_{10^{+3}}^{+3}$ & $2.24886614761_{10}-5$ \\
\hline 7 & $2.890000000_{10^{+2}}$ & $1.59921829887_{10}^{-6}$ \\
\hline 8 & $1.80000000010^{+1}$ & $\overline{9.9605291971010^{-8}}$ \\
\hline 9 & $1.00000000010^{+0}$ & $5.5336273317210^{-9}$ \\
\hline 10 & $0.000000000_{10}^{+0}$ & $\overline{0.00000} 000000_{10^{-0}}^{-0}$ \\
\hline
\end{tabular}

That is, $S=e^{x}, \lambda_{0}=1, \lambda_{n}=2(n \geq 1)$. We take $x=1$ and initial values (3.15) with $\nu=9$ and obtain the results given in Table 1.

The rightmost column in Table 1 is obtained by dividing the results of the middle column by

$$
\rho \doteq \frac{1}{e} \sum_{n=0}^{9} \lambda_{n} y_{n}^{(9)}=1.8071328986_{10}{ }^{+8} .
$$

When we take $N=5$, which means we want to compute the sequence $I_{0}(1), I_{1}(1), \ldots, I_{5}(1)$, we see that these quantities are computed with at least 10 correct decimal digits.

\subsection{Examples of hypergeometric functions and recursions}

We mention classes of functions of hypergeometric type that are of interest for applications and give a few details about their recursions.

\subsubsection{Bessel functions}

In the case of ordinary Bessel functions, we have the recurrence relation

$$
y_{n+1}-\frac{2 n}{z} y_{n}+y_{n-1}=0, \quad z \neq 0,
$$


with solutions

$$
f_{n}=J_{n}(z), \quad g_{n}=Y_{n}(z) .
$$

This is covered by (3.11), with

$$
a=1, \quad \alpha=0, \quad b=-\frac{2}{z}, \quad \beta=1 .
$$

In this case

$$
\frac{f_{n+1}}{f_{n}} \sim \frac{z}{2 n}, \quad \frac{g_{n+1}}{g_{n}} \sim \frac{2 n}{z} .
$$

The known asymptotic behavior of the Bessel functions reads

$$
f_{n} \sim \frac{1}{n !}\left(\frac{z}{2}\right)^{n}, \quad g_{n} \sim-\frac{(n-1) !}{\pi}\left(\frac{2}{z}\right)^{n}, \quad n \rightarrow \infty .
$$

Similar results hold for the modified Bessel functions, with recurrence relation

$$
y_{n+1}+\frac{2 n}{z} y_{n}-y_{n-1}=0, \quad z \neq 0,
$$

with solutions $I_{n}(z)$ (minimal) and $K_{n}(z)$ (dominant).

There is an extensive literature on the use of recursion for evaluating Bessel functions, with as pioneering paper [34]; see also [4, 97, 67].

\subsubsection{Kummer functions}

The Kummer functions (or confluent hypergeometric functions) ${ }_{1} F_{1}$ and $U$ do not satisfy the same recurrence relations, but by multiplying them with certain gamma functions they do. We assume $z>0$. An overview of the relevant recurrence relations can be found in [1, Chap. 13].

Recursion with respect to $a$. The functions

$$
\frac{\Gamma(a+n)}{\Gamma(a+n+1-c)}{ }_{1} F_{1}\left(\begin{array}{c}
a+n \\
c
\end{array} ; z\right) \text { and } \frac{\Gamma(a+n)}{\Gamma(a)} U(a+n, c, z)
$$

are respectively dominant and minimal.

Recursion with respect to $c$. The functions

$$
\frac{\Gamma(c-a+n)}{\Gamma(c+n)}{ }_{1} F_{1}\left(\begin{array}{c}
a \\
c+n
\end{array} ; z\right) \text { and } U(a, c+n, z)
$$

are respectively minimal and dominant. 
There are other interesting cases: recursion with respect to both $a$ and $c$, and recursion with respect to negative $n$. All the possible cases are analyzed in [99], where it is shown that the Kummer recurrences always have a minimal solution except for the case of recursion over $a$ when $z$ is real and positive (for $a \rightarrow-\infty$ ) or negative real (for $a \rightarrow+\infty$ ). See also [29] and [55, $\S 4.5 .1]$.

\subsubsection{Gauss hypergeometric functions}

The recursions for the functions

$$
{ }_{2} F_{1}\left(\begin{array}{c}
a+\epsilon_{1} n, b+\epsilon_{2} n \\
c+\epsilon_{3} n
\end{array} ; z\right),
$$

where $\epsilon_{j}=0, \pm 1$, not all equal to zero, and $z$ is complex are analyzed in $[48,52]$. Of the 27 nontrivial cases, only a limited set of these recursions need to be considered. This is because of several relations between contiguous Gauss functions. Among other results, in [52] it is shown that the function (3.28) is minimal around $z=0$ when $\epsilon_{3}>0$. An overview of the relevant recurrence relations can be found in [1, Chap. 15].

\subsubsection{Legendre functions}

For definitions and properties, see [1, Chap. 8] and [105, Chap. 8]. Legendre functions are special cases of Gauss hypergeometric functions, but the recursions need special attention. When $\Re z>0, z \notin(0,1], P_{\nu}^{\mu}(z)$ is the minimal solution of the recursion with respect to positive order $\mu ; Q_{\nu}^{\mu}(z)$ is dominant. Particular cases are toroidal functions and conical functions. The latter have the form $P_{-1 / 2+i \tau}^{\mu}(z), Q_{-1 / 2+i \tau}^{\mu}(z)$, which are real for $z>-1$ and real $\tau$ and $\mu$.

For recursion with respect to the degree $\nu, Q_{\nu}^{\mu}(z)$ is a minimal solution and $P_{\nu}^{\mu}(z)$ is dominant.

For further details on numerical aspects and algorithms we refer to [38, $39,40,41,53,56]$ and $[55, \S 12.3]$.

\subsubsection{Coulomb wave functions}

Information on these functions can be found in [1, Chap. 14]. Coulomb wave functions are special cases of the Kummer functions, and they can also be viewed as generalizations of Bessel functions. The regular function $F_{\lambda}(\eta, \rho)$ is the minimal solution with respect to increasing $\lambda$, while the irregular $G_{\lambda}(\eta, \rho)$ function is a dominant one. Algorithms based on recursions are 
discussed in [81]; in $[92,93]$ several types of series expansions are considered, with references to earlier algorithms.

\subsubsection{Parabolic cylinder functions}

For definitions and properties, see [1, Chap. 19]. The standard forms are $U(a, z)$ and $V(a, z)$, and, again, special cases of the Kummer functions. The function $U(a, x)$ is minimal in the forward $a$-recursion. For negative values of $a$ the situation is quite different, and for $|a|$ large enough $\left(a<<-z^{2} / 4\right)$, the solutions are neither minimal nor dominant. See [55, p. 102]. Algorithms using recursion can be found in [50, 51, 91, 98].

\section{Chebyshev expansions}

Chebyshev expansions are examples of convergent expansions, considered earlier, but because of their special properties they deserve a separate discussion.

Chebyshev polynomials of the first kind $T_{n}(x)$ have the nice property $T_{n}(\cos \theta)=\cos (n \theta)$, giving an equal ripple in the $\theta$-interval $[0, \pi]$ and in the $x$-interval $[-1,1]$. Because of their excellent convergence properties, Chebyshev expansions may replace convergent power series and divergent asymptotic expansions, or they may be used for filling the gap between the domains where convergent and asymptotic expansions can be used.

The standard Chebyshev expansion is of the form

$$
f(x)=\sum_{n=0}^{\infty}{ }^{\prime} c_{n} T_{n}(x), \quad-1 \leq x \leq 1,
$$

where the prime means that the first term is to be halved. Provided that the coefficients $c_{k}$ decrease in magnitude sufficiently rapidly, the error made by truncating the Chebyshev expansion after the terms $k=n$, that is,

$$
E_{n}(x)=\sum_{k=n+1}^{\infty} c_{k} T_{k}(x),
$$

will be given approximately by

$$
E_{n}(x) \doteq c_{n+1} T_{n+1}(x),
$$

that is, the error approximately satisfies the equioscillation property, which is happening in best-approximation (mini-max) methods. 


\subsection{Clenshaw's summation method}

There is a very simple algorithm due to Clenshaw [23] for evaluating the finite sum

$$
S_{n}(x)=\frac{1}{2} c_{0}+\sum_{k=1}^{n} c_{k} T_{k}(x),
$$

which is based on the recurrence relation

$$
x T_{k}(x)=\frac{1}{2}\left(T_{k+1}(x)+T_{|k-1|}(x)\right) .
$$

The algorithm computes a sequence $b_{1}, b_{2}, \ldots, b_{n+1}$ and starts with putting $b_{n+1}=0$ and $b_{n}=c_{n}$. Next,

$$
b_{k}=2 x b_{k+1}-b_{k+2}+c_{k}, \quad k=n-1, n-2, \ldots, 1 .
$$

Then, $S_{n}(x)=x b_{1}-b_{2}+\frac{1}{2} c_{0}$.

\subsection{Methods for obtaining the coefficients}

The coefficients $c_{n}$ in the expansion in (4.1) can be obtained in several ways, and we mention a few elements of the main methods. For details we refer to [55, Chap. 3].

\subsubsection{Tabled coefficients}

In the case that the function $f$ is an elementary or a one-variable special function, such as the error function erf $x$, the Bessel function $J_{0}(x)$, and so on, the coefficients can be obtained from tables, see [72]. Usually 20D accuracy of the coefficients is given. In [89] 30D coefficients are given for the error function and the complementary error function. Nowadays, computer algebra systems can be used to obtain tables of high precision coefficients.

\subsubsection{Discretizing the integral}

A numerical method uses discretization of the integral representation. That is,

$$
c_{k}=\frac{2}{\pi} \int_{-1}^{1} \frac{f(x) T_{k}(x)}{\sqrt{1-x^{2}}} d x=\frac{2}{\pi} \int_{0}^{\pi} f(\cos \theta) \cos (k \theta) d \theta,
$$

and discretization gives

$$
c_{k} \doteq \frac{2}{n} \sum_{j=0}^{n} \prime \prime f\left(\cos \frac{\pi j}{n}\right) \cos \frac{\pi k j}{n},
$$


where the primes mean that the first and last terms is are to be halved. This is a discrete cosine transform, which can be computed by methods based on the fast Fourier transform [110].

\subsubsection{Clenshaw's method}

This method can be used for functions satisfying a linear ordinary differential equations with polynomial coefficients of the form

$$
\sum_{k=0}^{m} p_{k}(x) f^{(k)}(x)=h(x),
$$

with $p_{k}$ polynomials and where the coefficients of the Chebyshev expansion of the function $h$ are known. The idea is as follows. Next to the expansion in (4.1), we introduce expansions for the relevant derivatives:

$$
f^{(s)}(x)=\sum_{n=0}^{\infty}{ }^{\prime} c_{n}^{(s)} T_{n}(x), \quad s=0,1,2, \ldots,
$$

and from known properties of the Chebyshev polynomials we have

$$
2 r c_{r}^{(s)}=c_{r-1}^{(s+1)}-c_{r+1}^{(s+1)}, \quad r \geq 1 .
$$

A next element in Clenshaw's method is to handle the powers of $x$ occurring in the differential equation satisfied by $f$. For this we need the relation (4.5) and formulas for higher powers of $x$ in the left-hand side. These can easily be obtained from the above one.

By substituting the expansion in (4.1) in the equation in (4.9) and using the formulas for the powers of $x$, a set of recursions for the coefficients $c_{r}^{(s)}$ can be obtained. Together with boundary values (or other known relations) this set of recursions can be solved numerically, and Clenshaw [24, 25] explained how this can be done by using a backward recursion method.

For example, the exponential function $y(x)=e^{a x}$ satisfies the differential equation $y^{\prime}=a y$. Substituting expansions following from (4.1) we obtain $c_{r}^{(1)}=a c_{r}$. Using this in the form $c_{r+2}^{(1)}=a c_{r+2}$ and using (4.11) we have the recursion for the coefficients:

$$
2(r+1) r c_{r+1}=a\left(c_{r}-c_{r+2}\right), \quad r \geq 0 .
$$

This is the recursion relation for the modified Bessel function $I_{r}(a)$, and so the $c_{r}$ is multiple of this function (the other modified Bessel function $K_{r}(a)$ 
being excluded because of its behavior for large $r$ ). The value $y(0)=1$ gives $c_{r}=I_{r}(a)$. This result is known because of the expansion

$$
e^{a \cos \theta}=\sum_{r=0}^{\infty} I_{r}(a) \cos (r \theta) .
$$

It is not at all needed that we know the solution in terms of a known function; for numerical purposes it is enough to have (4.12), and to use a backward recursion scheme, the Miller algorithm, as explained in $§ 3.3$.

However, several questions arise in this successful method. The recursion given in (4.12) is very simple, and we can find its exact solution. In more complicated recursion schemes obtained for the coefficients $c_{r}^{(s)}$ this information is not available. The scheme may be of large order and may have several solutions of which the asymptotic behavior is unknown. So, in general, we don't know if Clenshaw's method for differential equations computes the solution that we want, and if for the wanted solution the scheme is stable in the backward direction.

Clenshaw's method goes wrong in another simple example. Consider $y(x)=e^{a x+b x^{2}}$ with differential equation $y^{\prime}=(a+2 b x) y$. It is again easy to give a recursion scheme for the coefficients $c_{r}^{(s)}$. It reads (we use also (4.5))

$$
c_{r}^{(1)}=a c_{r}+b\left(c_{r+1}+c_{|r-1|}\right), \quad r \geq 0 .
$$

The coefficient $c_{r}^{(1)}$ can be eliminated simply by using this relation with $r$ replaced with $r+2$, and invoking (4.11). This gives

$$
2(r+1) c_{r+1}=a\left(c_{r}-c_{r+2}\right)+b\left(c_{r+1}-c_{r+3}\right), \quad r=0,1,2, \ldots .
$$

When applying a backward recursion algorithm for computing $c_{r}$ it appears that the solution does not give the requested function $y(x)$. The problem in this case is that there are two independent minimal solutions of the recurrence relation and one dominant solution, and straightforward application of Miller's backward algorithm explained in $\S 3.3$ gives a linear combination of the two minimal solutions. There are modifications of the Miller algorithm, which can be used for obtaining the requested solution.

In [49] a similar phenomenon has been discussed for the computation of the Weber function $W(a, x)$, solution of the equation $y^{\prime \prime}+\left(x^{2} / 4-a\right) y=0$. In that paper we describe a modification of Miller's algorithm in detail. See also [74] for an instability problem in Chebyshev expansions for special functions. 


\subsubsection{Known coefficients in terms of special functions}

As we have seen in (4.13), the coefficients in a Chebyshev expansion for the exponential function are known in terms of special functions. There are many other examples, also for higher transcendental functions. For example, we have (see [72, p. 37])

$$
\begin{aligned}
& J_{0}(a x)=\sum_{n=0}^{\infty} \epsilon_{n}(-1)^{n} J_{n}^{2}(a / 2) T_{2 n}(x), \\
& J_{1}(a x)=2 \sum_{n=0}^{\infty}(-1)^{n} J_{n}(a / 2) J_{n+1}(a / 2) T_{2 n+1}(x),
\end{aligned}
$$

where $-1 \leq x \leq 1$ and $\epsilon_{0}=1, \epsilon_{n}=2$ if $n>0$. The parameter $a$ can be any complex number. Similar expansions are available for $J$-Bessel functions of any complex order, in which the coefficients are ${ }_{1} F_{2}$-hypergeometric functions, and explicit recursion relations are available for computing the coefficients. For general integer order, the coefficients are products of two $J$-Bessel functions, as in (4.16). See again [72].

Another example is the expansion for the error function,

$$
e^{a^{2} x^{2}} \operatorname{erf}(a x)=\sqrt{\pi} e^{\frac{1}{2} a^{2}} \sum_{n=0}^{\infty} I_{n+\frac{1}{2}}\left(\frac{1}{2} a^{2}\right) T_{2 n+1}(x), \quad-1 \leq x \leq 1,
$$

in which the modified Bessel function is used. Again, $a$ can be any complex number.

The complexity of computing the coefficients of the expansions in (4.16) seems to be greater than the computation of the function that has been expanded. In some sense this is true, but the coefficients in (4.16), and those of many other examples for special functions, satisfy linear recurrence relations, and the coefficients satisfying such relations can usually be computed very efficiently by the backward recursion algorithm; see $\S 3.3$.

The expansions in (4.16) and (4.17) can be viewed as expansions near the origin. Other expansions are available that can be viewed as expansions at infinity, and these may be considered as alternatives for asymptotic expansions of special functions. For example, for the confluent hypergeometric $U$-functions we have the convergent expansion in terms of shifted Chebyshev polynomials $T_{n}^{*}(x)=T_{n}(2 x-1)$ :

$$
(\omega z)^{a} U(a, c, \omega z)=\sum_{n=0}^{\infty} C_{n}(z) T_{n}^{*}(1 / \omega),
$$


where

$$
z \neq 0, \quad|\operatorname{ph} z|<\frac{3}{2} \pi, \quad 1 \leq \omega \leq \infty .
$$

Furthermore, $a, 1+a-c \neq 0,-1,-2, \ldots$. When equalities hold for these values of $a$ and $c$, the Kummer $U$-function reduces to a Laguerre polynomial. This follows from

$$
U(a, c, z)=z^{1-c} U(1+a-c, 2-c, z)
$$

and

$$
U(-n, \alpha+1, z)=(-1)^{n} n ! L_{n}^{\alpha}(z), \quad n=0,1,2, \ldots
$$

The expansion (4.18) is given in [72, p. 25]. The coefficients can be represented in terms of generalized hypergeometric functions, in fact, Meijer $G$-functions, and they can be computed from the recurrence relation

$$
\frac{2 C_{n}(z)}{\epsilon_{n}}=2(n+1) A_{1} C_{n+1}(z)+A_{2} C_{n+2}(z)+A_{3} C_{n+3}(z),
$$

where $b=a+1-c, \epsilon_{0}=\frac{1}{2}, \epsilon_{n}=1(n \geq 1)$, and

$$
\begin{aligned}
& A_{1}=1-\frac{(2 n+3)(n+a+1)(n+b+1)}{2(n+2)(n+a)(n+b)}-\frac{2 z}{(n+a)(n+b)}, \\
& A_{2}=1-\frac{2(n+1)(2 n+3-z)}{(n+a)(n+b)} \\
& A_{3}=-\frac{(n+1)(n+3-a)(n+3-b)}{(n+2)(n+a)(n+b)} .
\end{aligned}
$$

For applying the backward recursion algorithm it is important to know that

$$
\sum_{n=0}^{\infty}(-1)^{n} C_{n}(z)=1, \quad|\operatorname{ph} z|<\frac{3}{2} \pi .
$$

This follows from

$$
\lim _{\omega \rightarrow \infty}(\omega z)^{a} U(a, c, \omega z)=1 \quad \text { and } \quad T_{n}^{*}(0)=(-1)^{n} .
$$

The standard backward recursion scheme (see $\S 3$ ) for computing the coefficients $C_{n}(z)$ works only for $|\operatorname{ph} z|<\pi$, and for $\operatorname{ph} z= \pm \pi$ a modification seems to be possible; see [72, p. 26].

Although the expansion in (4.18) converges for all $z \neq 0$ in the indicated sector, it is better to avoid small values of the argument of the $U$-function. 
Luke gives an estimate of the coefficients $C_{n}(z)$ of which the dominant factor that determines the speed of convergence is given by

$$
C_{n}(z)=\mathcal{O}\left(n^{2(2 a-c-1) / 3} e^{-3 n^{\frac{2}{3}} z^{\frac{1}{3}}}\right), \quad n \rightarrow \infty,
$$

and we see that large values of $\Re z^{1 / 3}$ improve the convergence.

The expansion in (4.18) can be used for all special cases of the Kummer $U$-function, that is, for Bessel functions (Hankel functions and $K$-modified Bessel function), for the incomplete gamma function $\Gamma(a, z)$, with special cases the complementary error function and exponential integrals. In [55, $\S 3.10]$ numerical coefficients are derived for expansions of the Airy function $\operatorname{Ai}(x)$ for $x \geq 7$ and for its derivative by using the expansion in (4.18).

\section{Quadrature methods}

We start with a simple example in which an oscillatory integral can be transformed into a stable representation. Consider the integral

$$
F(\lambda)=\int_{-\infty}^{\infty} e^{-t^{2}+2 i \lambda t} d t=\sqrt{\pi} e^{-\lambda^{2}}
$$

Taking $\lambda=10$ we get

$$
F(\lambda) \doteq 0.6593662990_{10}^{-43} .
$$

When we ask a well-known computer algebra system to do a numerical evaluation of the integral, without using the exact answer in (5.1) and with standard 10 digits accuracy, we obtain

$$
F(\lambda) \doteq 0.24_{10}^{-12} .
$$

We see that in this way this simple integral, with strong oscillations, cannot be evaluated correctly. Increasing the accuracy from 10 to 50 digits we obtain the answer

$$
F(\lambda) \doteq 0.65936629906_{10}^{-43}
$$

the first 8 digits being correct. Observe that we can shift the path of integration upwards until we reach the point $t=i \lambda$, the saddle point, and we write

$$
F(\lambda)=\int_{-\infty}^{\infty} e^{-(t-i \lambda)^{2}-\lambda^{2}} d t=e^{-\lambda^{2}} \int_{-\infty}^{\infty} e^{-s^{2}} d s
$$


Now the saddle point is at $s=0$, we integrate through this point along a path where no oscillations occur, a steepest descent path. Moreover the small factor $e^{-\lambda^{2}}$ that causes the main problems in the standard quadrature method, is now in front of the $s$-integral.

Similar methods can be applied to more complicated functions, in particular to a wide class of special functions from mathematical physics. Much software has been developed for many of these functions, but for large parameters the software is not at all complete and reliable, in particular when the parameters are large and complex.

We have come to the conclusion that methods based on asymptotic analysis are important for evaluating integrals by quadrature. Choosing suitable paths of integration and scaling the functions by separating dominant factors are important steps in these methods.

In this section we discuss the application of a simple quadrature rule, namely, the trapezoidal rule. For integral representations of special functions it may perform very well. Not always the standard integral representations should be taken, but modifications obtained by transformations or by choosing contours in the complex plane.

\subsection{The trapezoidal rule}

Gauss quadrature is a well-known quadrature method for evaluating integrals. It has a very good performance for various types of integrals over real intervals, given that the quadrature has maximal degree of exactness. However, one of the drawbacks is that it is not very flexible in algorithms when we want adjustable precision or when additional parameters are present. Also, we need zeros and weights of a certain class of orthogonal polynomials. For high precision algorithms computing these numbers in advance may be time consuming and/or not reliable.

The $n$-point extended trapezoidal rule

$$
\int_{a}^{b} f(t) d t=\frac{1}{2} h[f(a)+f(b)]+h \sum_{j=1}^{n-1} f(h j)+R_{n}, \quad h=\frac{b-a}{n},
$$

is more flexible, because we don't need precomputed zeros and weights; for this rule these numbers are trivial.

The error term has the form

$$
R_{n}=-\frac{1}{12}(b-a) h^{2} f^{\prime \prime}(\xi),
$$


Table 2: The remainder $R_{n}$ of the rule in (5.9) for several choices of $n$

\begin{tabular}{||r|l||}
\hline$n$ & $R_{n}$ \\
\hline 4 & $-.12_{10}^{-0}$ \\
8 & $-.48_{10^{-6}}$ \\
16 & $-.11_{10^{-21}}$ \\
32 & $-.13_{10^{-62}}$ \\
64 & $-.13_{10}^{-163}$ \\
128 & $-.53_{10}^{-404}$ \\
\hline
\end{tabular}

for some point $\xi \in(a, b)$, and for functions with continuous second derivative.

More insight in the error term follows from the Euler-Maclaurin summation rule [55, p. 131]. This rule gives the representation (for functions $f$ having $2 m+2$ continuous derivatives in $[a, b])$ :

$$
\begin{aligned}
R_{n}= & \sum_{j=0}^{m} \frac{B_{2 j}}{(2 j) !} h^{2 j}\left(f^{(2 j-1)}(a)-f^{(2 j-1)}(b)\right)- \\
& (b-a) h^{2 m+2} \frac{B_{2 m+2}}{(2 m+2) !} f^{(2 m+2)}(\xi),
\end{aligned}
$$

for some point $\xi \in(a, b) . B_{m}$ are the Bernoulli numbers. The first numbers with even index are $B_{0}=1, B_{2}=\frac{1}{6}, B_{4}=-\frac{1}{30}$.

We take as an example the Bessel function

$$
\pi J_{0}(x)=\int_{0}^{\pi} \cos (x \sin t) d t=h+h \sum_{j=1}^{n-1} \cos [x \sin (h j)]+R_{n},
$$

where $h=\pi / n$, and use this rule for $x=5$. The results of computations are shown in Table 2.

We observe that the error $R_{n}$ is much smaller than the upper bound that can be obtained from (5.7). The explanation comes from the periodicity in the integral for the Bessel function. Hence, all terms of the sum in (5.8) vanish, and we infer that now the error is $\mathcal{O}\left(h^{2 m+2}\right)$. And because for 
this integral this is true for any positive $m$, we conclude that the error is exponentially small as a function of $h$.

Another example is the Bessel function integral for general order

$$
J_{\nu}(x)=\frac{1}{2 \pi i} \int_{\mathcal{C}} e^{-x \sinh t+\nu t} d t
$$

where $\mathcal{C}$ starts at $-\infty-i \pi$ and terminates at $\infty+i \pi$; see [105, p. 222]. On this contour oscillations will occur, but we will select a special contour that is free of oscillations for the case $x \leq \nu$.

We write $\nu=x \cosh \mu, \mu \geq 0$. The saddle point of $-x \sinh t+\nu t=$ $-x(\sinh t-t \cosh \mu)$ occurs at $t=\mu$, and at this saddle point the imaginary part of $-x \sinh t+\nu t$ equals zero. A path free of oscillations (a steepest descent path through the saddle point) can be described by the equation $\Im(-x \sinh t+\nu t)=0$. Writing $t=\sigma+i \tau$ we obtain for the path the equation

$$
\cosh \sigma=\cosh \mu \frac{\tau}{\sin \tau}, \quad-\pi<\tau<\pi .
$$

On this path we have

$$
\Re(-x \sinh t+\nu t)=-x(\sinh \sigma \cos \tau-\sigma \cosh \mu) .
$$

Integrating with respect to $\tau$, using $d t / d \tau=(d \sigma / d \tau+i)$ (where $d \sigma / d \tau$ is an odd function of $\tau$ ), we obtain

$$
J_{\nu}(x)=\frac{1}{2 \pi} \int_{-\pi}^{\pi} e^{-x(\sinh \sigma \cos \tau-\sigma \cosh \mu)} d \tau, \quad 0<x \leq \nu .
$$

The integrand is analytic and vanishes with all its derivatives at the points $\pm \pi$. We can interpret the integrand as being a periodic $C^{\infty}$ function with period $2 \pi$, and consider representation (5.8) of the remainder. Again, the error in the trapezoidal rule is exponentially small.

When $\nu \gg x$ the Bessel function becomes very small and we can take the dominant part $e^{-x(\sinh \mu-\mu \cosh \mu)}$ in front of the integral. When $x \geq \nu$ (the oscillatory case), the Bessel function can be represented in a similar way, now by using two integrals (coming from the Hankel functions).

Our main conclusion of this section is that the trapezoidal rule may be very efficient and accurate when dealing with a certain class of integrals. Smoothness and periodicity of the integrand are the key properties here. 


\subsubsection{The trapezoidal rule on $\mathbb{R}$}

In the previous section we considered integrals over finite intervals. For integrals over $\mathbb{R}$ the trapezoidal rule may again be very efficient and accurate.

We consider

$$
\int_{-\infty}^{\infty} f(t) d t=h \sum_{j=-\infty}^{\infty} f(h j+d)+R_{d}(h),
$$

where $h>0$ and $0 \leq d<h$. We apply this rule with even functions $f$ analytic in a strip $G_{a}$ of width $2 a>0$ around $\mathbb{R}$ :

$$
G_{a}=\{z=x+i y \mid x \in \mathbb{R},-a<y<a\},
$$

which are bounded in $G_{a}$ and for which $\lim _{x \rightarrow \pm \infty} f(x+i y)=0$ (uniformly in $|y| \leq a)$ and

$$
M_{a}(f)=\int_{-\infty}^{\infty}|f(x+i a)| d x<\infty .
$$

Then, for real functions $f$, the remainder $R_{d}(h)$ of (5.14) satisfies

$$
\left|R_{d}(h)\right| \leq \frac{e^{-\pi a / h}}{\sinh (\pi a / h)} M_{a}(f) .
$$

The proof is based on residue calculus; see [55, p. 151].

As an example we consider the modified Bessel function

$$
K_{0}(x)=\frac{1}{2} \int_{-\infty}^{\infty} e^{-x \cosh t} d t
$$

We have, with $d=0$,

$$
e^{x} K_{0}(x)=\frac{1}{2} h+h \sum_{j=1}^{\infty} e^{-x(\cosh (h j)-1)}+R_{0}(h) .
$$

For $x=5$ and several values of $h$ we obtain the results given in Table $3\left(j_{0}\right.$ denotes the number of terms used in the series in (5.19)).

We see in this example that halving the value of $h$ gives a doubling of the number of correct significant digits (and, roughly speaking, a doubling of the number of terms needed in the series). When programming this method, observe that when halving $h$, previous function values can be used. 
Table 3: The remainder $R_{0}$ of the rule in (5.19) for several choices of $h$

\begin{tabular}{||l|r|l||}
\hline$h$ & $j_{0}$ & $R_{0}(h)$ \\
\hline 1 & 2 & $-.18_{10^{-1}}$ \\
$1 / 2$ & 5 & $-.24_{10^{-6}}$ \\
$1 / 4$ & 12 & $-.65_{10}^{-15}$ \\
$1 / 8$ & 29 & $-.44_{10}^{-32}$ \\
$1 / 16$ & 67 & $-.19_{10}^{-66}$ \\
$1 / 32$ & 156 & $-.55_{10^{-136}}$ \\
$1 / 64$ & 355 & $-.17_{10^{-272}}$ \\
\hline
\end{tabular}

\subsection{Complex contours}

In $\S 5.1$ we have transformed the complex contour integral (5.10) for the Bessel function $J_{\nu}(x)$ into a more suitable integral (5.13) by using saddle point methods. Here we explain how this works for the Airy function for applying the trapezoidal rule of on the real line of $\S 5.1 .1$. This gives a very flexible and efficient algorithm with adjustable precision.

We consider

$$
\operatorname{Ai}(z)=\frac{1}{2 \pi i} \int_{\mathcal{C}} e^{\frac{1}{3} w^{3}-z w} d w,
$$

where $\operatorname{ph} z \in\left[0, \frac{2}{3} \pi\right]$ and $\mathcal{C}$ is a contour starting at $\infty e^{-i \pi / 3}$ and terminating at $\infty e^{+i \pi / 3}$ (in the valleys of the integrand).

Let

$$
\phi(w)=\frac{1}{3} w^{3}-z w .
$$

The saddle points are $w_{0}=\sqrt{z}$ and $-w_{0}$ and follow from solving $\phi^{\prime}(w)=$ $w^{2}-z=0$.

The saddle point contour (the path of steepest descent) that runs through the saddle point $w_{0}$ is defined by

$$
\Im[\phi(w)]=\Im\left[\phi\left(w_{0}\right)\right] .
$$

We write

$$
z=x+i y=r e^{i \theta}, \quad w=u+i v, \quad w_{0}=u_{0}+i v_{0} .
$$


Figure 1: Saddle point contours for $\theta=0, \frac{1}{3} \pi, \frac{2}{3} \pi, \pi$ and $r=5$.

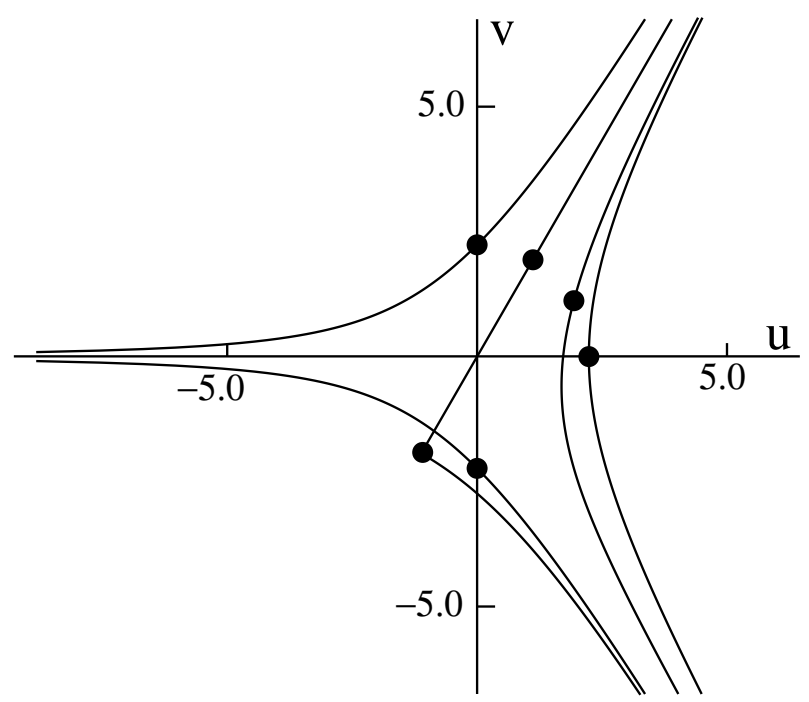

Then

$$
u_{0}=\sqrt{r} \cos \frac{1}{2} \theta, \quad v_{0}=\sqrt{r} \sin \frac{1}{2} \theta, \quad x=u_{0}^{2}-v_{0}^{2}, \quad y=2 u_{0} v_{0} .
$$

The path of steepest descent through $w_{0}$ is given by the equation

$$
u=u_{0}+\frac{\left(v-v_{0}\right)\left(v+2 v_{0}\right)}{3\left[u_{0}+\sqrt{\frac{1}{3}\left(v^{2}+2 v_{0} v+3 u_{0}^{2}\right)}\right]}, \quad-\infty<v<\infty .
$$

Examples for $r=5$ and a few $\theta$-values are shown in Figure 1. The relevant saddle points are located on the circle with radius $\sqrt{r}$ and are indicated by small dots.

The saddle point on the positive real axis corresponds with the case $\theta=0$ and the two saddles on the imaginary axis with the case $\theta=\pi$. This is out of the range of present interest, but it is instructive to see that the contour may split up and run through both saddle points $\pm w_{0}$. When $\theta=\frac{2}{3} \pi$ both saddle points are on one path, and the half-line in the $z$-plane corresponding with this $\theta$ is called a Stokes line (see $\S 6$ ).

Integrating with respect to $\tau=u-u_{0}$ (and writing $\sigma=v-v_{0}$ ) we obtain

$$
\operatorname{Ai}(z)=\frac{e^{-\zeta}}{2 \pi i} \int_{-\infty}^{\infty} e^{\psi_{r}(\sigma, \tau)}\left(\frac{d \sigma}{d \tau}+i\right) d \tau
$$


where $\zeta=\frac{2}{3} z^{\frac{3}{2}}$, and

$$
\begin{gathered}
\sigma=\frac{\tau\left(\tau+3 v_{0}\right)}{3\left[u_{0}+\sqrt{\frac{1}{3}\left(\tau^{2}+4 v_{0} \tau+3 r\right)}\right]}, \quad-\infty<\tau<\infty, \\
\psi_{r}(\sigma, \tau)=\Re\left[\phi(w)-\phi\left(w_{0}\right)\right]=u_{0}\left(\sigma^{2}-\tau^{2}\right)-2 v_{0} \sigma \tau+\frac{1}{3} \sigma^{3}-\sigma \tau^{2} .
\end{gathered}
$$

Quadrature methods for evaluating complex Airy functions can be found in $[36,43,44,45,90]$.

\section{The Stokes phenomenon}

The Stokes phenomenon concerns the abrupt change across certain rays in the complex plane, known as Stokes lines, exhibited by the coefficients multiplying exponentially subdominant terms in compound asymptotic expansions. There is much recent interest in the Stokes phenomenon, and it fits in the present paper because it has to do with sudden changes in approximations when a certain parameter (in this case the phase of the large parameter) passes critical values.

\subsection{The Airy function}

First we explain this phenomenon by using a simple example from differential equations. Consider Airy's equation

$$
\frac{d^{2} w}{d z^{2}}=z w
$$

the solutions of which are entire functions. When $|z|$ is large the solutions of (6.1) are approximated by linear combinations of

$$
w_{ \pm}=z^{-\frac{1}{4}} e^{ \pm \xi}, \quad \xi=\frac{2}{3} z^{3 / 2} .
$$

Obviously, $w_{ \pm}$are multivalued functions of the complex variable $z$ with a branch point at $z=0$. Therefore, as we go once around the origin, the solutions of (6.1) will return to their original values, but $w_{ \pm}$will not. It follows that the constants $c_{ \pm}$in the linear combination

$$
w(z) \sim c_{-} w_{-}(z)+c_{+} w_{+}(z), \quad z \rightarrow \infty,
$$


are domain-dependent. The constants change when we cross certain lines, the boundaries of certain sectors in the $z$-plane.

In the above example one of the terms $e^{\xi}, e^{-\xi}$ maximally dominates the other one at the rays $\operatorname{ph} z=0, \operatorname{ph} z= \pm 2 \pi / 3$. In this example these 3 rays are the Stokes lines. At the rays $\operatorname{ph} z= \pm \frac{1}{3} \pi$ and the negative $z$-axis the quantity $\xi$ is purely imaginary, and, hence, the terms $e^{\xi}, e^{-\xi}$ are equal in magnitude. These three rays are called the anti-Stokes lines ${ }^{4}$.

For the Airy function $\operatorname{Ai}(z)$ we have the full asymptotic expansion (see [1, Chap. 10])

$$
\operatorname{Ai}(z) \sim c_{-} z^{-\frac{1}{4}} e^{-\xi} \sum_{n=0}^{\infty}(-1)^{n} c_{n} \xi^{-n} \quad c_{-}=\frac{1}{2} \pi^{-\frac{1}{2}}, \quad|\mathrm{ph} z|<\pi,
$$

with coefficients

$$
c_{n}=\frac{\Gamma\left(3 n+\frac{1}{2}\right)}{54^{n} n ! \Gamma\left(n+\frac{1}{2}\right)}, \quad n=0,1,2, \ldots .
$$

On the other hand, in another sector of the $z$-plane, we have

$$
\operatorname{Ai}(-z) \sim c_{-} z^{-\frac{1}{4}}\left[e^{-\xi} \sum_{n=0}^{\infty}(-1)^{n} c_{n} \xi^{-n}+i e^{\xi} \sum_{n=0}^{\infty} c_{n} \xi^{-n}\right],
$$

in which exactly the same term (with the same constant $c_{-}$) is involved as in (6.4), and there is another term corresponding with $w_{+}$. We can rewrite this in a more familiar expansion

$$
\begin{aligned}
\operatorname{Ai}(-z) \sim \pi^{-\frac{1}{2}} z^{-\frac{1}{4}}( & \sin \left(\xi+\frac{1}{4} \pi\right) \sum_{n=0}^{\infty}(-1)^{n} \frac{c_{2 n}}{\xi^{2 n}}- \\
& \left.\cos \left(\xi+\frac{1}{4} \pi\right) \sum_{n=0}^{\infty}(-1)^{n} \frac{c_{2 n+1}}{\xi^{2 n+1}}\right)
\end{aligned}
$$

valid in the sector $|\mathrm{ph} z|<\frac{2}{3} \pi$. In the overlapping domain of expansions (6.4) and (6.7), that is, when $\frac{1}{3} \pi<|\mathrm{ph} z|<\pi$, the term with $w_{+}$is asymptotically small compared with $w_{-}$, and it suddenly appears in the asymptotic approximation when we cross with increasing values of $|\mathrm{ph} z|$ the Stokes lines at $\operatorname{ph} z= \pm \frac{2}{3} \pi$. It seems that, when going from (6.4) to (6.6), the constant

\footnotetext{
${ }^{4}$ This terminology is not the same in all branches of applied mathematics and mathematical physics: sometimes one sees a complete interchange of the names 'Stokes line' and 'anti-Stokes line'.
} 
multiplying $w_{+}$changes discontinuously from zero values (when $|\operatorname{ph} z|<\frac{2}{3} \pi$ ) to a non-zero value when we cross the Stokes line. This sudden appearance of the term $w_{+}$does not have much influence on the asymptotic behavior near the Stokes lines at $|\mathrm{ph} z|=\frac{2}{3} \pi$, because $w_{+}$is dominated maximally by $w_{-}$at these rays. However, see $\S 6.3$ below. Observe also the special value $\theta=\frac{2}{3} \pi$ in $\S 5.2$.

\subsection{The recent interest in the Stokes phenomenon}

This phenomenon of the discontinuity of the constants was discovered by Stokes and was discussed by him in a series of papers (on Airy functions in 1857, on Bessel functions in 1868). It is a phenomenon which is not confined to Airy or Bessel functions. The discovery by Stokes was, as Watson says, apparently one of those which are made at three o'clock in the morning. Stokes wrote in a 1902 retrospective paper: "The inferior term enters as it were into a mist, is hidden for a little from view, and comes out with its coefficients changed."

In 1989 the mathematical physicist Michael Berry provided a deeper explanation. He suggested that the coefficients of the subdominant expansion should be regarded not as a discontinuous constant but, for fixed $|z|$, as a continuous function of ph $z$. Berry's innovative and insightful approach was followed by a series of papers by himself and other writers. In particular, Olver put the formal approach by Berry on a rigorous footing in papers with applications to confluent hypergeometric functions (including Airy functions, Bessel functions, and Weber parabolic functions).

At the same time interest arose in earlier work by Stieltjes, Airey, Dingle, $\ldots$ to expand remainders of asymptotic expansions at optimal values of the summation variable. This resulted in exponentially-improved asymptotic expansions, a method of improving asymptotic approximations by including small terms in the expansion that are in fact negligible compared with other terms in the expansion.

\subsection{Exponentially small terms in the Airy expansions}

We conclude this discussion by pointing out the relation between the Stokes phenomenon and the exponentially small terms in the asymptotic expansion of the Airy function. Consider the terms in the expansions in (6.4)-(6.7). They have the asymptotic form

$$
c_{n} \xi^{-n}=\mathcal{O}\left[\Gamma(n)(2 \xi)^{-n}\right], \quad n \rightarrow \infty .
$$


When $z$ is large the terms decrease at first and then increase. The least term of the first series of (6.6) is near $n=n^{*}=[|2 \xi|]$ and its size is of order $e^{-2|\xi|}$. At the Stokes lines at $|\mathrm{ph} z|=\frac{2}{3} \pi$ the quantity $\xi$ is negative and the exponential term in front of the first series in (6.6) equals $e^{|\xi|}$. Hence the order of magnitude of $e^{-\xi} c_{n^{*}} \xi^{-n^{*}}$ is roughly of the same size as the second part in (6.7), that is, of the size of $e^{\xi}$ that is present in front of the second series. It follows that near the Stokes lines (and of course when $z$ turns to the negative axis) the second series in (6.7) is not at all negligible when we truncate the first series at the least term with index $n^{*}$.

At present we know, after Berry's observations, that near the Stokes lines one of the constants $c_{ \pm}$in the asymptotic representation in (6.2) in fact is a rapidly changing function of $z$. In the case of (6.6) we can write

$$
\operatorname{Ai}(z) \sim c_{-} z^{-\frac{1}{4}}\left[e^{-\xi} \sum_{n=0}^{\infty}(-1)^{n} c_{n} \xi^{-n}+i S(z) e^{\xi} \sum_{n=0}^{\infty} c_{n} \xi^{-n}\right],
$$

where $S(z)$ switches rapidly but smoothly from 0 to 1 across the Stokes line at $\mathrm{ph} z=\frac{2}{3} \pi$. A good approximation to $S(z)$ involves the error function, which function can describe the fast transition in this asymptotic problem.

Many writers have contributed recently in this field, both for the Stokes phenomenon of integrals and that of differential equations. For more details see the survey paper [83].

\section{A selection of other methods}

Many other methods are available for computing special functions. In this section we mention a selection. For all these topics more details can be found in [55].

\subsection{Continued fractions}

For many elementary and special functions representations as continued fractions exist. We give examples for incomplete gamma functions and incomplete beta functions that are useful for numerical computations.

We introduce the following notation. Let $\left\{a_{n}\right\}_{n=1}^{\infty}$ and $\left\{b_{n}\right\}_{n=0}^{\infty}$ be two sequences of real or complex numbers. With these numbers we construct a 
continued fraction of the form

$$
b_{0}+\frac{a_{1}}{b_{1}+\frac{a_{2}}{b_{2}+\frac{a_{3}}{b_{3}+\frac{a_{4}}{b_{4}+} \cdot}}}
$$

A more convenient notation is

$$
b_{0}+\frac{a_{1}}{b_{1}+} \frac{a_{2}}{b_{2}+} \frac{a_{3}}{b_{3}+} \frac{a_{4}}{b_{4}+\cdots} .
$$

For convergence aspects, contractions, even and odd parts, equivalence transformations, and so on, we refer to the literature; see [55, Chap. 6] and a recent handbook with many details for special functions [28].

To evaluate the finite part (also called the convergent)

$$
C_{n}=b_{0}+\frac{a_{1}}{b_{1}+} \frac{a_{2}}{b_{2}+} \frac{a_{3}}{b_{3}+} \cdots \frac{a_{n}}{b_{n}}
$$

we can use recursion. Let

$$
A_{-1}=1, \quad A_{0}=b_{0}, \quad B_{-1}=0, \quad B_{0}=1 .
$$

We compute $A_{n}$ and $B_{n}$ by using the following recursion

$$
A_{n}=b_{n} A_{n-1}+a_{n} A_{n-2}, \quad B_{n}=b_{n} B_{n-1}+a_{n} B_{n-2}, \quad n \geq 1 .
$$

Then $C_{n}=A_{n} / B_{n}$. Several other algorithms are available; see [55, §6.6]. The recursion for $A_{n}$ and $B_{n}$ may produce large numbers of these quantities, causing overflow. However, because only the ratio $A_{n} / B_{n}$ is needed to compute the convergent $C_{n}$, scaling can be used to keep control.

\subsubsection{Incomplete gamma functions}

The incomplete gamma functions are defined by

$$
\gamma(a, z)=\int_{0}^{z} t^{a-1} e^{-t} d t, \quad \Gamma(a, z)=\int_{z}^{\infty} t^{a-1} e^{-t} d t,
$$

where for the first form we require $\Re a>0$ and for the second one $|\operatorname{ph} z|<\pi$.

We have

$$
z^{-a} e^{z} \gamma(a, z)=b_{0}+\frac{a_{1}}{b_{1}+} \frac{a_{2}}{b_{2}+} \frac{a_{3}}{b_{3}+} \frac{a_{4}}{b_{4}+\cdots},
$$


where $z$ and $a$ are complex, $a \neq 0,-1,-2, \ldots$, and

$$
b_{0}=\frac{1}{a-z}, \quad a_{m}=m z, \quad b_{m}=a+m-z, \quad m \geq 1 .
$$

This fraction corresponds to the power series

$$
a z^{-a} e^{z} \gamma(a, z)=\sum_{k=0}^{\infty} \frac{z^{k}}{(1+a)_{k}} .
$$

For $\Gamma(a, z)$ we have

$$
(z+1-a) z^{-a} e^{z} \Gamma(a, z)=\frac{1}{1+} \frac{\alpha_{1}}{1+} \frac{\alpha_{2}}{1+} \frac{\alpha_{3}}{1+\cdots},
$$

where

$$
\alpha_{n}=\frac{n(a-n)}{(z+2 n-1-a)(z+2 n+1-a)}, \quad n=1,2,3, \ldots .
$$

This form is used in [35] for computing the function $\Gamma(a, x)$, for $x>1.5$ and $-\infty<a<\alpha^{*}(z)$, where $\alpha^{*}(x) \sim x$ for large $x$. The fraction in (7.10) is convergent for all $z \neq 0$ in the sector $|\mathrm{ph} z|<\pi$, and for computations it is an excellent alternative for the corresponding asymptotic expansion

$$
z^{1-a} e^{z} \Gamma(a, z) \sim \sum_{k=0}^{\infty}(-1)^{k} \frac{(1-a)_{k}}{z^{k}},
$$

valid for $a \in \mathbb{C}, z \rightarrow \infty,|\operatorname{ph} z|<\frac{3}{2} \pi$.

\subsubsection{Incomplete beta function}

This function is defined by

$$
B_{x}(p, q)=\int_{0}^{x} t^{p-1}(1-t)^{q-1} d t, \quad \Re p>0, \quad \Re q>0,
$$

and usually $0 \leq x \leq 1$; when $x<1$ the condition on $q$ can be omitted. The beta integral is obtained when we take $x=1$, that is,

$$
B(p, q)=\int_{0}^{1} t^{p-1}(1-t)^{q-1} d t=\frac{\Gamma(p) \Gamma(q)}{\Gamma(p+q)}, \quad \Re p>0, \quad \Re q>0 .
$$

We have the continued fraction

$$
p x^{-p}(1-x)^{-q} B_{x}(p, q)=\frac{1}{1+} \frac{d_{1}}{1+} \frac{d_{2}}{1+} \frac{d_{3}}{1+\cdots},
$$


where, for $n=0,1,2, \ldots$,

$$
\begin{aligned}
& d_{2 n+1}=-\frac{(p+n)(p+q+n)}{(p+2 n)(p+2 n+1)} x, \\
& d_{2 n+2}=\frac{(n+1)(q-n-1)}{(p+2 n+1)(p+2 n+2)} x .
\end{aligned}
$$

When $p>1$ and $q>1$, the maximum of the integrand in (7.13) occurs at $x_{0}=(p-1) /(p+q-2)$, and the best numerical results are obtained when $x \leq x_{0}$. When $x_{0}<x \leq 1$, we use the reflection relation with the beta integral (see (7.14))

$$
B_{x}(p, q)=B(p, q)-B_{1-x}(q, p) .
$$

From a numerical point of view the continued fraction (7.15) has an interesting property of the convergents: $C_{4 n}$ and $C_{4 n+1}$ are less than this value of the continued fraction and $C_{4 n+2}, C_{4 n+3}$ are greater than this value. This gives excellent control of the convergence of an algorithm that uses (7.15).

\subsection{Sequence transformations}

When applying numerical techniques to physical problems, results are usually produced in the form of sequences. Examples are iterative methods, discretization methods, perturbation methods, and - most important in the context of special functions - series expansions. Often, the sequences that are produced in this way converge too slowly to be numerically useful. When dealing with asymptotic series, summation of the sequences may also be difficult.

Sequence transformations are tools to overcome convergence problems of that kind. A slowly convergent (or even divergent in the asymptotic sense) sequence $\left\{s_{n}\right\}_{n=0}^{\infty}$, whose elements may be the partial sums

$$
s_{n}=\sum_{k=0}^{n} a_{k}
$$

of a convergent or formal infinite series, is converted into a new sequence $\left\{s_{n}^{\prime}\right\}_{n=0}^{\infty}$ with hopefully better numerical properties.

We discuss sequence transformations that are useful in the context of special functions. For many special functions convergent and divergent (asymptotic) power series are available. Consequently, the emphasis in this section 
will be on sequence transformations that are able either to accelerate the convergence of slowly convergent power series effectively or to sum divergent asymptotic series.

\subsubsection{Padé approximations}

From a numerical point of view, the Padé approximants are important for computing functions outside the disk of convergence of the power series of the function, as well as inside the disk (for example, near the boundary of the disk). The Padé method can also be successfully applied for locating zeros and poles of the function.

Consider the power series

$$
f(z)=c_{0}+c_{1} z+c_{2} z^{2}+\cdots,
$$

with $c_{0} \neq 0$. This series may be convergent or just a formal power series. We introduce a rational function $N_{m}^{n}(z) / D_{m}^{n}(z)$, where $N_{m}^{n}(z)$ and $D_{m}^{n}(z)$ are polynomials of maximal degree $n$ and $m$, respectively. That is,

$$
N_{m}^{n}(z)=a_{0}+a_{1} z+\cdots+a_{n} z^{n}, \quad D_{m}^{n}(z)=b_{0}+b_{1} z+\cdots+b_{m} z^{m} .
$$

We choose these polynomials such that the power series expansion of $N_{m}^{n}(z)-$ $f(z) D_{m}^{n}(z)$ starts with a term $A_{n, m} z^{n+m+1}$. The ratio $N_{m}^{n}(z) / D_{m}^{n}(z)$, of which the polynomials $N_{m}^{n}(z)$ and $D_{m}^{n}(z)$ satisfy the conditions

$$
\begin{aligned}
& \text { degree } N_{m}^{n}(z) \leq n, \quad \text { degree } D_{m}^{n}(z) \leq m, \\
& N_{m}^{n}(z)-f(z) D_{m}^{n}(z)=A_{n, m} z^{n+m+1}+\cdots,
\end{aligned}
$$

is called a Padé approximant of type $(n, m)$ to the power series (7.19) (the function $f)$. The ratio $N_{m}^{n}(z) / D_{m}^{n}(z)$ is denoted by $[n / m]_{f}$.

For each pair $(n, m)$ at least one rational function exists that satisfies the conditions in (7.21), and this function can be found by solving the equations

$$
\left\{\begin{array} { l } 
{ a _ { 0 } = c _ { 0 } b _ { 0 } , } \\
{ a _ { 1 } = c _ { 1 } b _ { 0 } + c _ { 0 } b _ { 1 } , } \\
{ \quad \vdots } \\
{ a _ { n } = c _ { n } b _ { 0 } + c _ { n - 1 } b _ { 1 } + \cdots + c _ { n - m } b _ { m } , }
\end{array} \left\{\begin{array}{c}
0=c_{n+1} b_{0}+\cdots+c_{n-m+1} b_{m}, \\
\vdots \\
0=c_{n+m} b_{0}+\cdots+c_{n} b_{m},
\end{array}\right.\right.
$$


where $c_{j}=0$ if $j<0$. When $m=0$ the system of equations at the right is empty. In this case $a_{j}=c_{j}(j=0,1, \ldots, n)$ and $b_{0}=1$, and the partial sums of (7.19) yield the Padé approximants of type $(n, 0)$. In general, first the set at the right-hand side of (7.22) is solved (a homogeneous set of $m$ equations for the $m+1$ values $b_{j}$ ), which has at least one nontrivial solution. We take a normalization, for example, by taking $b_{0}=1$ (see also the discussion in $\left[7\right.$, p. 18]), and with this choice the last $m$ equations give $b_{1}, \ldots, b_{m}$ as the solution of a system of $m$ linear equations. The set on the left-hand side in (7.22) then yields $a_{0}, \ldots, a_{n}$.

The array of Padé approximants

$$
\begin{array}{cccc}
{[0 / 0]_{f}} & {[0 / 1]_{f}} & {[0 / 2]_{f}} & \cdots \\
{[1 / 0]_{f}} & {[1 / 1]_{f}} & {[1 / 2]_{f}} & \cdots \\
{[2 / 0]_{f}} & {[2 / 1]_{f}} & {[2 / 2]_{f}} & \cdots
\end{array}
$$

is called a Padé table. It is arranged here so that approximants with the same denominator degree are located in the same column. As remarked earlier, the first column corresponds to the partial sums of the power series in (7.19). The elements of the first row correspond to the partial sums of the power series of $1 / f$.

In the literature special attention is paid to the diagonal elements $[n, n]_{f}$ of the table, with applications to orthogonal polynomials, quadrature formulas, moment problems, and other problems of classical analysis.

In applied mathematics and in theoretical physics, Padé approximants have become a useful tool for overcoming convergence problems with power series. The popularity of Padé approximants in theoretical physics is due to Baker [5], who also wrote a monograph on Padé approximants [6]. Of interest also is the monograph by Baker and Graves-Morris [7].

An extended bibliography on Padé approximants and related matters containing several thousand references was published by Brezinski in [13]. For an annotated bibliography focusing on computational aspects, see [119]. Luke gives many rational approximations of special functions, and usually these are Padé approximants; see [72, 73].

\subsubsection{How to compute the Padé approximants}

The approximants can be computed by Wynn's cross rule. Any five Padé approximants arranged in the Padé table as 
satisfy Wynn's cross rule (see [121])

$$
(N-C)^{-1}+(S-C)^{-1}=(W-C)^{-1}+(E-C)^{-1} .
$$

Starting with the first column $[n / 0]_{f}, n=0,1,2, \ldots$, initializing the preceding column by $[n /-1]_{f}=\infty, n=1,2, \ldots,(7.24)$ enables us to compute the lower triangular part of the table. Likewise, the upper triangular part follows from the first row $[0 / n]_{f}, n=0,1,2, \ldots$, by initializing $[-1 / n]_{f}=0$, $n=1,2, \ldots$.

The elements of the Padé table can also be computed by the epsilon algorithm of Wynn [120]. We consider the recursions

$$
\begin{aligned}
& \varepsilon_{-1}^{(n)}=0, \quad \varepsilon_{0}^{(n)}=s_{n}, \quad n=0,1,2, \ldots \\
& \varepsilon_{m+1}^{(n)}=\varepsilon_{m-1}^{(n+1)}+\frac{1}{\varepsilon_{m}^{(n+1)}-\varepsilon_{m}^{(n)}}, \quad n, m=0,1,2, \ldots
\end{aligned}
$$

If $s_{n}$ is the $n$th partial sum of a power series $f$, then $\varepsilon_{2 k}^{(n)}$ is the Padé approximant $[n+k / k]_{f}$ (cf. $\left.(7.23)\right)$. The elements $\varepsilon_{2 k+1}^{(n)}$ are only auxiliary quantities which diverge if the whole transformation process converges and shouldn't be used for convergence tests or output. A recent review of the applications of the epsilon algorithm can be found in [60].

In applications one usually concentrates on obtaining diagonal elements $[n / n]_{f}$ and elements not far away from the diagonal; see [114], which also has an efficient modified algorithm for these elements.

\subsubsection{Nonlinear sequence transformations}

We discuss a few other sequence transformations that, in the case of power series, produce different rational approximants, and they can also be applied to other convergence acceleration problems.

Details on the history of sequence transformations and related topics, starting from the 17th century, can be found in [14]; see also [15]. For review papers, with many references to monographs devoted to this topic, we refer the reader to $[62,114]$. See also Appendix A in [11], written by Dirk Laurie, with interesting observations and opinions about sequence transformations. 
First we mention Levin's sequence transformation [68], which is defined by

$$
\mathcal{L}_{k}^{(n)}\left(s_{n}, \omega_{n}\right)=\frac{\sum_{j=0}^{k}(-1)^{j}\left(\begin{array}{l}
k \\
j
\end{array}\right) \frac{(n+j+1)^{k-1}}{(\zeta+n+k)^{k-1}} \frac{s_{n+j}}{\omega_{n+j}}}{\sum_{j=0}^{k}(-1)^{j}\left(\begin{array}{l}
k \\
j
\end{array}\right) \frac{n+j+1)^{k-1}}{(\zeta+n+k)^{k-1}} \frac{1}{\omega_{n+j}}},
$$

where $s_{n}$ are the partial sums of (7.18) and the quantities $\omega_{n}$ are remainder estimates. For example, we can simply take $\zeta=1$ and

$$
\omega_{n}=s_{n+1}-s_{n}=a_{n+1},
$$

but more explicit remainder estimates can be used.

Another transformation is due to Weniger [114], who replaced the powers $(n+j+1)^{k-1}$ in Levin's transformation by Pochhammer symbols $(n+j+$ $1)_{k-1}$. That is, Weniger's transformation reads

$$
\mathcal{S}_{k}^{(n)}\left(s_{n}, \omega_{n}\right)=\frac{\sum_{j=0}^{k}(-1)^{j}\left(\begin{array}{l}
k \\
j
\end{array}\right) \frac{(\zeta+n+j)_{k-1}}{(\zeta+n+k)_{k-1}} \frac{s_{n+j}}{\omega_{n+j}}}{\sum_{j=0}^{k}(-1)^{j}\left(\begin{array}{l}
k \\
j
\end{array}\right) \frac{(\zeta+n+j)_{k-1}}{(\zeta+n+k)_{k-1}} \frac{1}{\omega_{n+j}}} .
$$

Other sequence transformations can be found in [114] or in [16, §2.7]. The sequence transformations (7.26) and (7.28) differ from other sequence transformations because not only the elements of a sequence $\left\{s_{n}\right\}$ are required, but also explicit remainder estimates $\left\{\omega_{n}\right\}$. For special functions this information is usually available when divergent asymptotic expansions are considered. It was shown in several articles that the transformation (7.28) is apparently very effective, in particular if divergent asymptotic series are to be summed; see $[9,118,116]$.

For transforming partial sums $f_{n}(z)=\sum_{k=0}^{n} \gamma_{k} z^{k}$ of a formal power series

$$
f(z)=\sum_{k=0}^{\infty} \gamma_{k} z^{k},
$$

we can take the remainder estimates

$$
\omega_{n}=\gamma_{n+1} z^{n+1},
$$

and we replace $z$ by $1 / z$ in the case of an asymptotic series. 
With these modifications the transformations (7.26) and (7.28) become rational functions of the variable $z$. If the coefficients $\gamma_{n}$ in (7.29) are all different from zero, these rational functions satisfy the asymptotic error estimates [117, eqs. (4.28)-(4.29)]

$$
\begin{aligned}
& f(z)-\mathcal{L}_{k}^{(n)}\left(f_{n}(z), \gamma_{n+1} z^{n+1}\right)=\mathcal{O}\left(z^{k+n+2}\right), \quad z \rightarrow 0, \\
& f(z)-\mathcal{S}_{k}^{(n)}\left(f_{n}(z), \gamma_{n+1} z^{n+1}\right)=\mathcal{O}\left(z^{k+n+2}\right), \quad z \rightarrow 0 .
\end{aligned}
$$

These estimates imply that all terms of the formal power series, which were used for construction of the rational approximants in this way, are reproduced exactly by a Taylor expansion around $z=0$. Thus, the transformations $\mathcal{L}_{k}^{(n)}\left(f_{n}(z), \gamma_{n+1} z^{n+1}\right)$ and $\mathcal{S}_{k}^{(n)}\left(f_{n}(z), \gamma_{n+1} z^{n+1}\right)$ are formally very similar to the analogous estimate (7.21) satisfied by the Padé approximants $[n / m]_{f}(z)=N_{m}^{n}(z) / D_{m}^{n}(z)$.

\subsubsection{Numerical examples}

Simple test problems, which nevertheless demonstrate convincingly the power of sequence transformations using explicit remainder estimates, are the integrals

$$
\mathbf{E}^{(\nu)}(z)=\int_{0}^{\infty} \frac{\mathrm{e}^{-t} d t}{1+z t^{\nu}}
$$

and their associated divergent asymptotic expansions

$$
\mathbf{E}^{(\nu)}(z) \sim \sum_{k=0}^{\infty}(\nu k) !(-z)^{k}, \quad z \rightarrow 0
$$

For $\nu=1, \mathbf{E}^{(\nu)}(z)$ is the exponential integral $E_{1}$ with argument $1 / z$ according to $\mathbf{E}^{(1)}(z)=\mathrm{e}^{1 / z} E_{1}(1 / z) / z$. For $\nu=2$ or $\nu=3, \mathbf{E}^{(\nu)}(z)$ cannot be expressed in terms of known special functions.

In order to demonstrate the use of sequence transformations with explicit remainder estimates, both $\mathcal{S}_{k}^{(n)}\left(f_{n}(z), \gamma_{n+1} z^{n+1}\right)$ and Padé approximants are applied to the partial sums

$$
E_{n}^{(\nu)}(z)=\sum_{k=0}^{n}(\nu k) !(-z)^{k}, \quad 0 \leq n \leq 50
$$

of the asymptotic series (7.33) for $\nu=1,2,3$. The Padé approximants were computed with the help of Wynn's epsilon algorithm (see §7.2.2). All 
calculations were done in Maple, and the integrals $\mathbf{E}^{(\nu)}(z)$ were computed to the desired precision with the help of numerical quadrature. For the remainder estimates we took $\omega_{n}=(\nu(n+1)) !(-z)^{n+1}$.

The results for $\mathbf{E}^{(1)}(z)$ with $z=1$ are

$$
\begin{aligned}
\mathbf{E}^{(1)}(1) & =0.5963473623231940743410785, \\
\mathcal{L}_{0}^{(50)}\left(E_{0}^{(1)}(1), \omega_{50}\right) & =0.5963473623231940743410759, \\
\mathcal{S}_{0}^{(50)}\left(E_{0}^{(1)}(1), \omega_{50}\right) & =0.5963473623231940743410785, \\
{[25 / 24] } & =0.596347322, \\
{[25 / 25] } & =0.596347387 .
\end{aligned}
$$

The Padé approximants are not very efficient. Nevertheless, it seems that they are able to sum the divergent series (7.33) for $\nu=1$.

The results for $\mathbf{E}^{(2)}(z)$ with $z=1 / 10$ are

$$
\begin{aligned}
\mathbf{E}^{(2)}(1 / 10) & =0.884251306126979, \\
\mathcal{L}_{0}^{(50)}\left(E_{0}^{(2)}(1 / 10), \omega_{50}\right) & =0.884251306126980, \\
\mathcal{S}_{0}^{(50)}\left(E_{0}^{(2)}(1 / 10), \omega_{50}\right) & =0.884251306126985, \\
{[25 / 24] } & =0.88409, \\
{[25 / 25] } & =0.88437 .
\end{aligned}
$$

Here, the Padé approximants are certainly not very useful since they can only extract an accuracy of three places.

The results for $\mathbf{E}^{(3)}(z)$ with $z=1 / 100$ are

$$
\begin{aligned}
\mathbf{E}^{(3)}(1 / 100) & =0.9620671061, \\
\mathcal{S}_{0}^{(50)}\left(E_{0}^{(3)}(1 / 100), \omega_{50}\right) & =0.9620671055, \\
\mathcal{L}_{0}^{(50)}\left(E_{0}^{(3)}(1 / 100), \omega_{50}\right) & =0.9620671057, \\
{[25 / 24] } & =0.960, \\
{[25 / 25] } & =0.964 .
\end{aligned}
$$

In [59] it is shown that an asymptotic series, whose coefficients grow more rapidly than $(2 n)$ !, is not Padé summable since subsequences $[n+j / n]$ in the 
Padé table converge to different, $j$-dependent limits as $n \rightarrow \infty$. The Levin and Weniger transformations are apparently able to sum the asymptotic series (7.33) even for $\nu=3$.

Other numerical examples of sequence transformations using explicit remainder estimates can be found in $[9,115,116]$. For a recent paper on transformations of hypergeometric series, see [21].

\subsection{Other quadrature methods}

In $\S 5$ we have described the use of the trapezoidal rule in the evaluation of special functions. In $[55, \S 5.3, \S 9.6]$ several other methods are discussed, with as the main one Gaussian quadrature, and the relation with orthogonal polynomials.

Other methods are Romberg quadrature, which provides a scheme for computing successively refined rules with a higher degree of exactness. Fejér and Clenshaw-Curtis quadratures are interpolatory rules which behave quite similarly to Gauss-Legendre rules, but which are easier to compute and provide nested rules. Other nested rules, related to Gauss quadrature but harder to compute than the Clenshaw-Curtis rule, are Kronrod and Patterson quadratures. Specific methods for oscillatory integrands are also described, with special attention to Filon's method.

\subsection{Numerical inversion of Laplace transforms}

We consider the pair of Laplace transforms

$$
F(s)=\int_{0}^{\infty} e^{-s t} f(t) d t, \quad f(t)=\frac{1}{2 \pi i} \int_{c-i \infty}^{c+i \infty} e^{s t} F(s) d s,
$$

where $f$ should be absolutely integrable on any finite interval $[0, a]$ and the number $c$ is chosen such that all singularities of $F(s)$ are at the left of the vertical line $\Re s=c$.

The inversion problem is to find $f(t)$ when $F(s)$ is given. To solve this problem numerically, an essential condition is whether function values of $F(s)$ are only available for real $s$ or for complex values of $s$. The first case is quite difficult and requires completely different techniques compared with those for the second case. In this section we consider a method for the case that $F(s)$ is available as an analytic function in part of the complex $s$-plane. We describe a method based on the deformation of the contour of integration. 
We give an example in which an optimal representation of the integral is obtained by deforming the contour of integration and by using a proper value of $c$ in the complex integral in (7.38). After selecting this new contour, the trapezoidal rule can be used for numerical quadrature. As explained in $\S 5$ this method may be very efficient for evaluating a class of integrals with analytic integrands.

We use the Laplace transform pair (see [1, eq. (29.3.83)])

$$
\begin{aligned}
& F(s)=\frac{1}{s} e^{-k \sqrt{s}}=\int_{0}^{\infty} e^{-s t} \operatorname{erfc} \frac{k}{2 \sqrt{t}} d t, \\
& \operatorname{erfc} \frac{k}{2 \sqrt{t}}=\frac{1}{2 \pi i} \int_{c-i \infty}^{c+i \infty} e^{s t-k \sqrt{s}} \frac{d s}{s},
\end{aligned}
$$

where in this case $c>0$. We take $k=2 \lambda$ and $t=1$, which gives

$$
\operatorname{erfc} \lambda=\frac{1}{2 \pi i} \int_{c-i \infty}^{c+i \infty} e^{s-2 \lambda \sqrt{s}} \frac{d s}{s}
$$

and we assume that $\lambda>0$. When $\lambda$ is large the integral becomes exponentially small, and straightforward application of a quadrature rule is useless.

With the transformation $s=\lambda^{2} t,(7.40)$ becomes

$$
\operatorname{erfc} \lambda=\frac{1}{2 \pi i} \int_{c-i \infty}^{c+i \infty} e^{\lambda^{2}(t-2 \sqrt{t})} \frac{d t}{t} \text {. }
$$

When we take $c=1$ the path runs through the saddle point at $t=1$, where the exponential function of the integrand has the value $e^{-\lambda^{2}}$, which corresponds to the main term in the asymptotic estimate

$$
\operatorname{erfc} \lambda \sim \frac{e^{-\lambda^{2}}}{\sqrt{\pi} \lambda}, \quad \lambda \rightarrow \infty .
$$

Because the convergence at $\pm i \infty$ along the vertical through $t=1$ is rather poor, the next step is to deform the contour into a new contour that terminates in the left half-plane, with $\Re t \rightarrow-\infty$.

In fact many contours are suitable, but there is only one contour through $t=1$ on which no oscillations occur. That contour, the steepest descent path, is given by $\Im(t-2 \sqrt{t})=0$, or in polar coordinates $t=r e^{i \theta}$ we have $r=\sec ^{2}\left(\frac{1}{2} \theta\right)$. See Figure 2. This gives, by integrating with respect to $\theta \in[-\pi, \pi]$,

$$
\operatorname{erfc} \lambda=\frac{e^{-\lambda^{2}}}{2 \pi} \int_{-\pi}^{\pi} e^{-\lambda^{2} \tan ^{2}\left(\frac{1}{2} \theta\right)} d \theta
$$




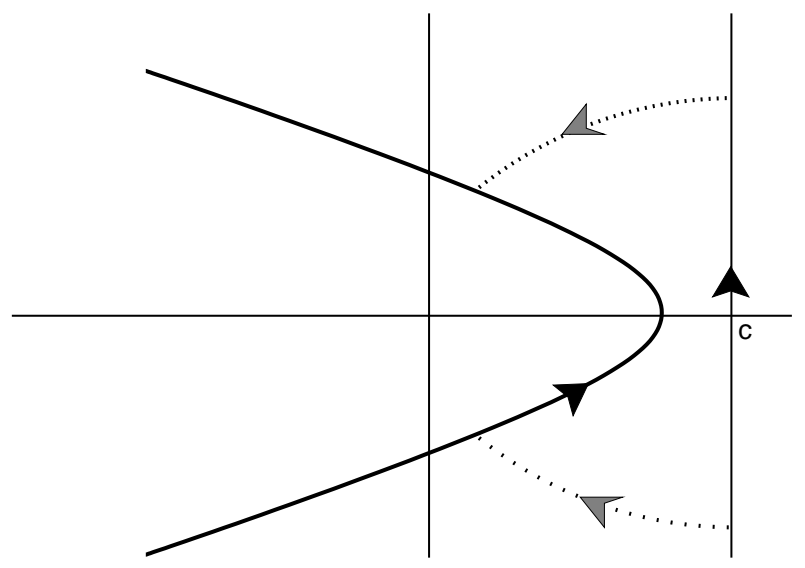

Figure 2: The new contour of integration for (7.41) has the shape of a parabola.

As discussed in $\S 5.1$ the trapezoidal rule is exceptionally accurate in this case.

Table 4 gives the results of applying the composite trapezoidal rule with step size $h ; n$ indicates the number of function values in the rule that are larger than $10^{-15}$ (we exploit the fact that the integrand is even). All digits shown in the approximation in the final row are correct.

When $F(s)$ in (7.38) has singularities or poles, a straightforward and optimal choice of the path of integration, as in the above example, might not be easy to find. In these cases, or when less information is available on the function $F(s)$, a less optimal contour may be chosen.

Table 4: Composite trapezoidal rule for the integral in (7.43) with $\lambda=10$.

\begin{tabular}{||c|c|r||}
\hline$h$ & $\operatorname{erfc} \lambda$ & $n$ \\
\hline 0.25 & $0.209494943296679_{10}-44$ & 5 \\
0.20 & $0.208861164534559_{10}{ }^{-44}$ & 6 \\
0.15 & $0.208848758872946_{10}^{-44}$ & 8 \\
0.10 & $0.208848758376254_{10}^{-44}$ & 11 \\
\hline
\end{tabular}



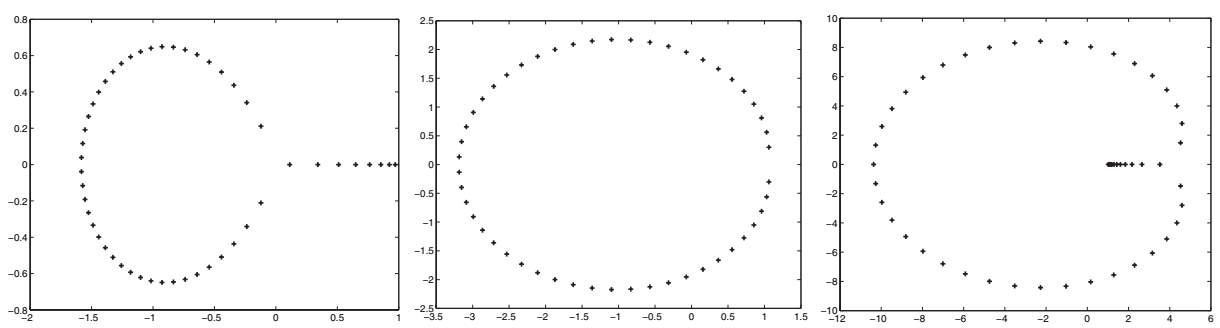

Figure 3: Zeros of the Jacobi polynomials $P_{50}^{(2,-42.5)}(x)$ (left), $P_{50}^{(2,-52)}(x)$ (center), and $P_{50}^{(2,-63.5)}(x)$ (right) in the complex plane.

For example, we can take a parabola or a hyperbola that terminates in the left half-plane at $-\infty$. When we write $s=u+i v$, and consider the parabola defined by $u=p-q v^{2}$, and integrate with respect to $v$. When we choose $p$ and $q$ properly all singularities of $F(s)$ may remain inside the contour (unless $F(s)$ has an infinite number of singularities up to $\pm i \infty$ ).

Further details can be found in $[80,87,101]$. Recent investigations are discussed in $[88,108,112,113]$.

\subsection{Computing zeros of special functions}

The zeros of special functions appear in a great number of applications in mathematics, physics, and engineering, from the computation of Gauss quadrature rules [57] in the case of orthogonal polynomials to many applications in which boundary value problems for second order ordinary differential equations arise.

In some sense, the computation of the zeros of special functions has nothing special: to compute the roots of an equation $y(x)=0$, with $y(x)$ special or not, we can apply well-known methods (bisection, secant, NewtonRaphson, or whatever) once we know how to compute the function $y(x)$ (and in the case of Newton-Raphson also its derivative) accurately enough.

However, as is generally the case with nonlinear equations, some information on the location of the zeros is desirable, particularly when applying rapidly convergent (but often unpredictable) methods like Newton-Raphson or higher order methods.

The zeros of special functions usually appear nicely arranged, forming clear patterns from which a priori information can be found. For instance, the zeros of Jacobi polynomials $P_{n}^{(\alpha, \beta)}(x)$ are all real and in the interval $(-1,1)$ for $\alpha>-1$ and $\beta>-1$, and they satisfy other regularity properties (such as, for instance, interlacing with the zeros of the derivative and with 
the zeros of contiguous orders). As $\alpha$ and/or $\beta$ become smaller than -1 , some or all of the $n$ zeros escape from the real axis, forming a regular pattern in the complex plane (see Figure 3).

These regular patterns formed by the zeros is a common feature of "classical" special functions and beyond [22]. The regularity in the distribution of zeros helps in the design of specific algorithms with good convergence properties. In addition, for many special functions, accurate a priori approximations are available. This a priori information, when wisely applied, will save computation time and avoid divergent (or even chaotic) algorithms. In a fortunate situation, as in the case of Bessel functions, asymptotic approximations provide accurate enough starting values for higher order NewtonRaphson methods; see [104].

In [55, Chap. 7], a variety of methods for computing zeros of special functions is discussed, starting with bisection and the fixed point method, including Newton-Raphson. In general, for computing zeros of special functions it is a wise idea to use some of their properties and to design specific methods.

Next to methods that take advantage of information about asymptotic approximations for the zeros of special functions, there are methods for which it is not necessary to compute values of these functions themselves in order to obtain their zeros. This is the case for the classical orthogonal polynomials, the zeros of which are the exact eigenvalues of real tridiagonal symmetric matrices with very simple entries; this method is usually named the Golub-Welsch algorithm [57]. The recurrence relation of the special functions plays a crucial role because the matrix is built from the coefficients of the recursion. Also, there are other functions, minimal solutions of threeterm recurrence relations (the Bessel function $J_{\nu}(x)$ is among them) for which the problem of computing zeros is not exactly an eigenvalue problem for a (finite) matrix, but it can be approximated by it [58, 64].

Another type of methods, which are global in the sense that, similarly as matrix methods, don't require a priori estimations of the zeros for ensuring convergence, are the fixed point methods of $[42,96]$; these methods use first order differential systems, which are satisfied by a large number of special functions (hypergeometric functions among them). More recently, a fixed order method of fourth order was obtained in [95], which can be used to compute the zeros of any solution of any second order equation $y^{\prime \prime}(x)+A(x) y(x)=0$ in an interval where $A(x)$ is continuous. It is shown 
that, when $A(x)>0$, the fixed point iteration

$$
T(x)=x-\frac{1}{\sqrt{A(x)}} \arctan \left(\sqrt{A(x)} y(x) / y^{\prime}(x)\right)
$$

has order four and the sequence $x_{n+1}=T\left(x_{n}\right)$ converges for any $x_{0}$ under mild conditions on $A(x)$. A scheme is given which guarantees convergence for any continuous $A(x)$ and allows for computing all the zeros in an interval. Typically 3 or 4 iterations per zero are required for 100 digits accuracy.

Additional methods and details on obtaining information on the zeros by using asymptotic expansions of the special functions, with examples such as Airy functions, Scorer functions, error functions, parabolic cylinder functions, Bessel functions, and Laguerre polynomials $L_{n}^{(\alpha)}(x)$ with large values of $\alpha$ are given in [55, Chap. 7].

\subsection{Uniform asymptotic expansions}

The asymptotic expansions considered in $\S 2.1$ are simple in the sense that they hold for large values of one variable, in fact the argument of the special function. There are many powerful expansions available that hold also for other large parameters.

For example, the expansion for the incomplete gamma function in (7.12) holds when $z$ is large, but it becomes useless when $a$ is also large. Also, the expansion in (7.9) converges for all $a$ and $z$ with trivial exceptions: $a \neq 0,-1,-2, \ldots$. But for computations it becomes useless when $z$ is much larger than $a$.

There is a nice alternate asymptotic representations for these functions which can be used when $a$ and/or $z$ are large, and which in particular holds when $a \sim z$, a transition point in the behavior for large $a$ and $z$. In this representation the complementary error function

$$
\operatorname{erfc} z=\frac{2}{\sqrt{\pi}} \int_{z}^{\infty} e^{-t^{2}} d t
$$

plays the role of the transition form very small to very large values, or for the scaled functions

$$
P(a, z)=\frac{\gamma(a, z)}{\Gamma(a)}, \quad Q(a, z)=\frac{\Gamma(a, z)}{\Gamma(a)}
$$

from values close to 0 to values close to 1 . 
We have the following representations:

$$
\begin{aligned}
& Q(a, z)=\frac{1}{2} \operatorname{erfc}(\eta \sqrt{a / 2})+R_{a}(\eta), \\
& P(a, z)=\frac{1}{2} \operatorname{erfc}(-\eta \sqrt{a / 2})-R_{a}(\eta),
\end{aligned}
$$

where

$$
\frac{1}{2} \eta^{2}=\lambda-1-\ln \lambda, \quad \lambda=\frac{z}{a},
$$

and

$$
R_{a}(\eta)=\frac{e^{-\frac{1}{2} a \eta^{2}}}{\sqrt{2 \pi a}} S_{a}(\eta), \quad S_{a}(\eta) \sim \sum_{n=0}^{\infty} \frac{C_{n}(\eta)}{a^{n}}
$$

as $a \rightarrow \infty$.

The relation between $\eta$ and $\lambda$ in (7.48) becomes clear when we expand

$$
\lambda-1-\ln \lambda=\frac{1}{2}(\lambda-1)^{2}-\frac{1}{3}(\lambda-1)^{3}+\frac{1}{4}(\lambda-1)^{4}+\cdots,
$$

and in fact the relation in (7.48) can also be written as

$$
\eta=(\lambda-1) \sqrt{\frac{2(\lambda-1-\ln \lambda)}{(\lambda-1)^{2}}},
$$

where the sign of the square root is positive for $\lambda>0$. For complex values we use analytic continuation. An expansion for small values of $|\lambda-1|$ reads

$$
\eta=(\lambda-1)-\frac{1}{3}(\lambda-1)^{2}+\frac{7}{36}(\lambda-1)^{3}+\cdots,
$$

and, upon inverting this expansion,

$$
\lambda=1+\eta+\frac{1}{3} \eta^{2}+\frac{1}{36} \eta^{3}+\cdots .
$$

The asymptotic expansion for $S_{a}(\eta)$ in (7.49) holds uniformly with respect to $z \geq 0$. Both $a$ and $z$ may be complex. Note that the symmetry relation $P(a, z)+Q(a, z)=1$ is preserved in the representations in (7.47) because $\operatorname{erfc} z+\operatorname{erfc}(-z)=2$.

The first coefficients for $S_{a}(\eta)$ are

$$
C_{0}=\frac{1}{\lambda-1}-\frac{1}{\eta}, \quad C_{1}(\eta)=\frac{1}{\eta^{3}}-\frac{1}{(\lambda-1)^{3}}-\frac{1}{(\lambda-1)^{2}}-\frac{1}{12(\lambda-1)} .
$$

These coefficients, and all higher ones, are regular at the transition point $a=z$, or $\lambda=1$, or $\eta=0$. For numerical applications Taylor expansions can be used, as explained in $[55, \S 8.3]$. 
For Bessel functions we have a similar problem in the design of efficient algorithms. All Bessel functions can be expanded in terms of Airy functions, and these expansions are in particular useful in the neighborhood of the turning point $z=\nu$. For example, the Bessel function $J_{\nu}(z)$ is oscillatory for $z>\nu$ and monotonic for $z<\nu$. Airy functions have a similar turning point behavior, as follows from their differential equation $w^{\prime \prime}-z w=0$.

The coefficients in the asymptotic series are regular at the turning point $z=\nu$, but for numerical evaluations we need expansions of the used coefficients in the neighborhood of the turning point. For more details we refer to $[55, \S 8.4]$ and $[106]$.

Airy-type expansions are used in software for Bessel functions in $[4,46$, $67]$, and for parabolic cylinder functions in [49, 50, 91].

\subsection{Taylor expansion methods for ordinary differential equa- tions}

The special functions of mathematical physics usually arise as special solutions of ordinary linear differential equations, which follow from certain forms of the wave equation. Separation of the variables and the use of domains such as spheres, circles, cylinders, and so on, are the standard ways of introducing Bessel functions, Legendre functions, and confluent hypergeometric functions (also called Whittaker functions). For an introduction to this topic, see [105, Chap. 10].

In numerical mathematics, computing solutions of ordinary linear differential equations is a vast research area, with popular methods such as, for example, Runge-Kutta methods. These techniques are usually not used for computing special functions, mainly because so many other efficient methods are available for these functions. However, when the differential equation has coefficients in terms of analytic functions, as is the case for the equations of special functions, a method based on Taylor expansions may be considered as an alternative method, in particular for solving the equation in the complex plane.

In $[55, \S 9.5]$ the basic steps are given for the Taylor expansion method, in particular for linear second order equations of the form

$$
\frac{d^{2} w}{d z^{2}}+f(z) \frac{d w}{d z}+g(z) w=h(z),
$$

where $f, g$, and $h$ are analytic functions in a domain $D \subset \mathbb{C}$. For applications to special functions $f, g$, and $h$ are often simple rational functions, and usually the equation is homogeneous. 
For further information and examples, see [82] and [71]. For an application to compute solutions in the complex plane of the Airy differential equation, see [32] with a Fortran computer program in [31]. In [49] Taylor methods are used for computing the parabolic cylinder function $W(a, x)$.

\subsection{Computing symmetric elliptic integrals}

Legendre's standard elliptic integrals are the incomplete elliptic integral of the first kind,

$$
K(k)=\int_{0}^{\phi} \frac{d \theta}{\sqrt{1-k^{2} \sin ^{2} \theta}},
$$

the incomplete integral of the second kind,

$$
E(k)=\int_{0}^{\phi} \sqrt{1-k^{2} \sin ^{2} \theta} d \theta,
$$

and the incomplete elliptic integral of the third kind,

$$
\Pi(n ; \phi, k)=\int_{0}^{\phi} \frac{1}{1-n \sin ^{2} \theta} \frac{d \theta}{\sqrt{1-k^{2} \sin ^{2} \theta}} .
$$

It is assumed here that $k \in[0,1], \phi \in\left[0, \frac{1}{2} \pi\right]$, although the functions can also be defined for complex values of the parameters. Also, $n$ is real, and if $n>1$, the integral of the third kind should be interpreted as a Cauchy principal value integral. When $\phi=\frac{1}{2} \pi$ the integrals are called complete elliptic integrals.

The computational problem for the elliptic integrals has received much attention in the literature, and the algorithms are usually based on successive Landen transformations or Gauss transformations, or by infinite series.

By considering a new set of integrals it is possible to compute the elliptic integrals, also by using successive transformations, by very efficient algorithms. The integrals are introduced in [17]. For example we have

$$
R_{F}(x, y, z)=\frac{1}{2} \int_{0}^{\infty} \frac{d t}{\sqrt{(t+x)(t+y)(t+z)}} .
$$

This function is symmetric and homogeneous of degree $-\frac{1}{2}$ in $x, y, z$ and is normalized so that $R_{F}(x, x, x)=x^{-\frac{1}{2}}$.

Many elementary functions can be expressed in terms of these integrals. For example,

$$
R_{F}(x, y, y)=\frac{1}{2} \int_{0}^{\infty} \frac{d t}{\sqrt{(t+x)}(t+y)},
$$


which is a logarithm if $0<y<x$ and an inverse circular function if $0 \leq x<$ $y$.

The three standard elliptic integrals in (7.56)-(7.58) can be written in terms of symmetric integrals. For example, we have

$$
F(\phi, k)=\sin \phi R_{F}\left(\cos ^{2} \phi, 1-k^{2} \sin ^{2} \phi, 1\right) .
$$

For further details we refer to $[55, \S 11.4]$ and to the work of B.C. Carlson, who wrote very efficient algorithms for the computation of Legendre's standard elliptic integrals, also for complex parameters $[18,19,20]$.

\subsection{Best rational approximations}

In the theory of best rational approximation (which includes the best polynomial of approximation) the goal is to find a rational function that approximates a function $f$ on a finite real interval as best as possible. The rational approximations can be written in the form

$$
\frac{N_{m}^{n}(x)}{D_{m}^{n}(x)}=\frac{a_{0}+a_{1} x+\cdots+a_{n} x^{n}}{b_{0}+b_{1} x+\cdots+b_{m} x^{m}}
$$

The characterization of the best approximation to a function $f$ may be given in terms of oscillations of the error curve. Let $R=N / D$ be an irreducible rational function of the form (7.62). A necessary and sufficient condition that $R$ be the best approximation to $f$ is that the error function $R(x)-f(x)$ exhibits at least $2+\max \{m+\partial N, n+\partial D\}$ points of alternation. (Here $\partial P$ denotes the degree of the polynomial $P$.) For the proof see [76].

For the elementary and well-known higher transcendental functions the polynomials in the best rational approximations are not explicitly known, and the coefficients of these polynomials should be computed by an algorithm. This algorithm is not as simple as the one for computing coefficients in Chebyshev series (see §4) or Padé approximants (which can be based on solving a set of linear equations). For best approximation the second algorithm of Remes can be used [86, p. 176], and for a Fortran program see [66].

For many elementary and special functions best rational approximations have been computed. See [61] for many tables (and an explanation of the Remes algorithm). For several other special functions we refer to the survey [26]. Computer algebra packages, such as Maple, also have programs for computing best rational approximants.

For flexible algorithms for special functions we prefer the method based on Chebyshev polynomials. Chebyshev series (4.1) usually converge rapidly 
(for example, for functions in $C^{\infty}$ on $[-1,1]$ ), we obtain a very good first approximation to the polynomial $p_{n}(x)$ of best approximation for $[-1,1]$ if we truncate $(4.1)$ at its $(n+1)$ th term. This is because

$$
f(x)-\sum_{k=0}^{n}{ }^{\prime} c_{k} T_{k}(x) \doteq c_{n+1} T_{n+1}(x),
$$

and the right-hand side enjoys exactly those properties concerning its maxima and minima that are required for the polynomial of best approximation. In practice the gain in replacing a truncated Chebyshev series expansion by the corresponding minimax polynomial approximation is hardly worthwhile; see [84].

Inspection of the size of the coefficients $c_{k}$ gives a good idea about the applicability for a certain choice of $n$, and for a new choice of $n$ the computations are easy to modify. In best approximations for each choice of $n$ (or of $n$ and $m$ in rational approximations), new coefficients have to be computed by using a complicated algorithm. In addition, representations of the polynomials in best approximations may be quite unstable.

\section{Recent software and publications on methods for computing special functions}

\subsection{A selection of recent software for special functions}

Software packages as Mathematica, Maple, and Matlab have many excellent algorithms in multi-length arithmetic. For large scale and high performance computing these packages are not the optimal platforms. Also, there are many published books with software for special functions, some with supplied sources of the algorithms. We mention [8, 79, 85, 107, 111, 122]. Many software collections on special functions are available at the web, for instance the Cephes math library ${ }^{5}$ and in more general repositories. ${ }^{6} 7$

For an extensive survey of the available software for special functions we refer to [70]. The latest update of this project appeared in December 2000. In this section we give a selection of software for special functions published in the period 2000-2009 (disclaimer: we provide references, but we don't claim that all the software listed is equally reliable).

\footnotetext{
${ }^{5}$ http://www.moshier.net

${ }^{6}$ http://www.netlib.org/

${ }^{7}$ http://gams.nist.gov/Classes.html
} 


\section{Books describing published software}

1. Cuyt et al. [28], a Handbook describing numerical methods for evaluating special functions by using continued fractions. Many tables are given based on Maple programs published elsewhere by the group. All kinds of functions are considered: from gamma to Gauss, confluent, generalized and basic hypergeometric functions.

2. Gautschi [37] describes routines for generating recursion coefficients of orthogonal polynomials as well as routines dealing with applications.

3. Gil et al. [55] describes software for Airy and Scorer functions, associated Legendre functions of integer and half-integer degrees (including toroidal harmonics), Bessel functions (including modified Bessel functions with purely imaginary orders), parabolic cylinder functions, and a module for computing zeros of Bessel functions.

\section{Gamma, error and related functions}

1. Smith [100]: Fortran 90 software for floating-point multiple precision arithmetic, gamma and related functions.

2. Linhart et al. [69]: the logarithm of the normal distribution.

\section{Bessel functions}

1. Kodama [67]: all kinds of cylindrical functions of complex order and nonnegative argument.

2. Gil et al. [47]: modified Bessel functions $I_{i a}(x)$ and $K_{i a}(x)$ for real $a$ and positive $x$.

3. Van Deun and Cools [109]: infinite range integrals of an arbitrary product of Bessel functions.

4. Talman [102]: spherical Bessel transforms.

\section{Airy and related functions}

1. Gil et al. [44, 54]: complex Airy and Scorer functions.

2. Fabijonas [31]: complex Airy functions.

\section{Parabolic cylinder functions}


1. Gil et al. [50]: functions $U(a, x)$ and $V(a, x)$ for real $a$ and $x$.

\section{Coulomb wave functions}

1. Michel [77]: functions $F_{\ell}(\eta, \rho)$ and $G_{\ell}(\eta, \rho)$ for complex parameters

2. Seaton [93]: functions $F_{\ell}(\eta, \rho)$ and $G_{\ell}(\eta, \rho)$.

3. Seaton [94]: Numerov integrations of Coulomb functions.

4. Noble [81]: negative energy Coulomb (Whittaker) functions.

\section{Legendre functions}

1. Gil and Segura [40, 41]: toroidal harmonics.

2. Inghoff et al. [65]: Maple procedures for the coupling of angular momenta.

\section{Hypergeometric functions}

1. Michel and Stoitsov [78]: Gauss hypergeometric function with all its parameters complex.

2. Colavecchia and Gasaneo [27]: Appell's $F_{1}$ function.

3. Huber and Maître [63]: expanding hypergeometric functions about half-integer parameters.

\section{Mathieu functions}

1. Alhargan [2, 3]: Mathieu functions and characteristic numbers.

2. Erricolo [30]: expansion coefficients of Mathieu functions using Blanch's algorithm.

\subsection{Recent literature on the computation of special functions}

From our website ${ }^{8}$ a list will be soon available with recent literature from the last ten years (2000-2009). The list can be viewed as an addition to the bibliography of Lozier and Olver [70], and contains references to software and papers describing methods for computing special functions. Some of the references are mentioned in earlier sections. The following topics can be found in the list.

\footnotetext{
${ }^{8}$ http://functions.unican.es
} 
1. General aspects in books and papers: continued fractions, recurrence relations, Hadamard-type expansions, infinite products.

2. Gamma function, Barnes multiple gamma function, incomplete gamma functions, beta distribution, error functions, exponential integrals.

3. Bessel functions, integrals of Bessel functions, series of $K$-Bessel function.

4. Airy functions, Airy-type integrals: oscillatory cuspoid integrals with odd and even polynomial phase functions, Pearcy integral.

5. Hypergeometric functions: Gauss, confluent, Coulomb, Weber parabolic, Appell.

6. Legendre functions: toroidal, conical, spherical harmonics.

7. Orthogonal polynomials, Gauss quadrature.

8. $q$-functions.

9. Mathieu functions.

10. Spheroidal wave functions.

11. Polylogarithms.

12. Mittag-Leffler function, Wright function.

13. Elliptic integrals, elliptic functions.

14. Riemann zeta function, Riemann theta function.

15. Bose-Einstein, Fermi-Dirac integrals.

16. Hubbell rectangular source integrals, Lambert's $W$-function, leaky aquifer function.

17. Multicenter integrals, Slater orbitals, other integrals from physics.

18. Zeros of special functions.

19. Multiprecision implementation of elementary and special functions. 


\section{Acknowledgements}

The authors would like to thank Dr. Ernst Joachim Weniger for providing us with notes that we used for writing $\S 7.2$. The authors acknowledge financial support from Ministerio de Educación y Ciencia, project MTM200609050. NMT acknowledges financial support from Gobierno of Navarra, Res. 07/05/2008.

\section{References}

[1] M. Abramowitz and I. A. Stegun. Handbook of mathematical functions with formulas, graphs, and mathematical tables, volume 55 of National Bureau of Standards Applied Mathematics Series. US Printing Office, 1964.

[2] Fayez A. Alhargan. Algorithm 804: subroutines for the computation of Mathieu functions of integer orders. ACM Trans. Math. Software, 26(3):408-414, 2000.

[3] Fayez A. Alhargan. Algorithm 855: subroutines for the computation of Mathieu characteristic numbers and their general orders. ACM Trans. Math. Software, 32(3):472-484, 2006.

[4] D. E. Amos. Algorithm 644: a portable package for Bessel functions of a complex argument and nonnegative order. ACM Trans. Math. Software, 12(3):265-273, 1986.

[5] G. A. Baker, Jr. The theory and application of the Padé approximant method. In Advances in Theoretical Physics, Vol. 1, pages 1-58. Academic Press, New York, 1965.

[6] G. A. Baker, Jr. Essentials of Padé approximants. Academic Press [A subsidiary of Harcourt Brace Jovanovich, Publishers], New York-London, 1975.

[7] G. A. Baker, Jr. and P. Graves-Morris. Padé approximants, volume 59 of Encyclopedia of Mathematics and its Applications. Cambridge University Press, Cambridge, second edition, 1996.

[8] L. Baker. C Mathematical Function Handbook. Programming Tools For Engineers and Scientists. McGraw-Hill, Inc., New York, 1992.

[9] R. Bhattacharya, D. Roy, and S. Bhowmick. Rational interpolation using Levin-Weniger transforms. Comput. Phys. Comm., 101(3):213-222, 1997.

[10] W. G. Bickley, L. J. Comrie, J. C. P. Miller, D. H. Sadler, and A. J. Thompson. Bessel functions. Part II. Functions of positive integer order. British Association for the Advancement of Science, Mathematical Tables, vol. X. University Press, Cambridge, 1952. 
[11] F. Bornemann, D. Laurie, S. Wagon, and J. Waldvogel. The SIAM 100digit challenge. Society for Industrial and Applied Mathematics (SIAM), Philadelphia, PA, 2004. A study in high-accuracy numerical computing, With a foreword by David H. Bailey.

[12] John P. Boyd. The devil's invention: Asymptotic, superasymptotic and hyperasymptotic series. Acta Appl. Math., 56(1):1-98, 1999.

[13] C. Brezinski. A bibliography on continued fractions, Padé approximation, sequence transformation and related subjects. Prensas Universitarias de Zaragoza, Zaragoza, 1991.

[14] C. Brezinski. History of continued fractions and Padé approximants. SpringerVerlag, Berlin, 1991.

[15] C. Brezinski. Convergence acceleration during the 20th century. J. Comput. Appl. Math, 0:000-000, 2000. To appear.

[16] C. Brezinski and M. Redivo-Zaglia. Extrapolation methods. Theory and practice, volume 2 of Studies in Computational Mathematics. North-Holland Publishing Co., Amsterdam, 1991.

[17] B. C. Carlson. Special functions of applied mathematics. Academic Press [Harcourt Brace Jovanovich Publishers], New York, 1977.

[18] B. C. Carlson. Computing elliptic integrals by duplication. Numer. Math., $33(1): 1-16,1979$

[19] B. C. Carlson. Numerical computation of real or complex elliptic integrals. Numer. Algorithms, 10(1-2):13-26, 1995. Special functions (Torino, 1993).

[20] B. C. Carlson and James FitzSimons. Reduction theorems for elliptic integrals with the square root of two quadratic factors. J. Comput. Appl. Math., 118(12):71-85, 2000.

[21] Sanjukta Chatterjee and Dhiranjan Roy. A class of new transforms tailored for the hypergeometric series. Comput. Phys. Comm., 179(8):555-561, 2008.

[22] P. A. Clarkson and E. L. Mansfield. The second Painlevé equation, its hierarchy and associated special polynomials. Nonlinearity, 16(3):R1-R26, 2003.

[23] C. W. Clenshaw. A note on the summation of Chebyshev series. Math. Tables Aids Comput., 9(51):118-120, 1955.

[24] C. W. Clenshaw. The numerical solution of linear differential equations in Chebyshev series. Proc. Cambridge Philos. Soc., 53:134-149, 1957.

[25] C. W. Clenshaw. Chebyshev series for mathematical functions. National Physical Laboratory Mathematical Tables, Vol. 5. Department of Scientific and Industrial Research. Her Majesty's Stationery Office, London, 1962.

[26] W. J. Cody. A survey of practical rational and polynomial approximation of functions. SIAM Rev., 12(3):400-423, 1970. 
[27] F. D. Colavecchia and G. Gasaneo. f1: a code to compute Appell's $F_{1}$ hypergeometric function. Comput. Phys. Commun., 157(1):32-38, 2004.

[28] Annie Cuyt, Vigdis Brevik Petersen, Brigitte Verdonk, Haakon Waadeland, and William B. Jones. Handbook of continued fractions for special functions. Springer, New York, 2008. With contributions by Franky Backeljauw and Catherine Bonan-Hamada, Verified numerical output by Stefan Becuwe and Cuyt.

[29] Alfredo Deaño, Javier Segura, and Nico M. Temme. Identifying minimal and dominant solutions for Kummer recursions. Math. Comp., 77(264):2277-2293, 2008.

[30] Danilo Erricolo. Algorithm 861: Fortran 90 subroutines for computing the expansion coefficients of Mathieu functions using Blanch's algorithm. ACM Trans. Math. Software, 32(4):622-634, 2006.

[31] B. R. Fabijonas. Algorithm 838: Airy functions. ACM Trans. Math. Software, 30(4):491-501, 2004.

[32] B. R. Fabijonas, D. W. Lozier, and F. W. J. Olver. Computation of complex Airy functions and their zeros using asymptotics and the differential equation. ACM Trans. Math. Softw., 30(4):471-490, 2004.

[33] R. C. Forrey. Computing the hypergeometric function. J. Comput. Phys., 137(1):79-100, 1997.

[34] W. Gautschi. Computational aspects of three-term recurrence relations. SIAM Rev., 9(1):24-82, 1967.

[35] W. Gautschi. A computational procedure for incomplete gamma functions. ACMTM, 5:466-481, 1979.

[36] W. Gautschi. Computation of Bessel and Airy functions and of related Gaussian quadrature formulae. BIT, 42(1):110-118, 2002.

[37] W. Gautschi. Orthogonal polynomials: computation and approximation. Numerical Mathematics and Scientific Computation. Oxford University Press, New York, 2004. Oxford Science Publications.

[38] A. Gil and J. Segura. Evaluation of Legendre functions of argument greater than one. Comput. Phys. Comm., 105(2-3):273-283, 1997.

[39] A. Gil and J. Segura. A code to evaluate prolate and oblate spheroidal harmonics. Comput. Phys. Commun., 108(2-3):267-278, 1998.

[40] A. Gil and J. Segura. Evaluation of toroidal harmonics. Comput. Phys. Comm., 124:104-122, 2000.

[41] A. Gil and J. Segura. DTORH3 2. 0: A new version of a computer program for the evaluation of toroidal harmonics. Comput. Phys. Commun., 139(2):186191, 2001. 
[42] A. Gil and J. Segura. Computing the zeros and turning points of solutions of second order homogeneous linear ODEs. SIAM J. Numer. Anal., 41(3):827$855,2003$.

[43] A. Gil, J. Segura, and N. M. Temme. On nonoscillating integrals for computing inhomogeneous Airy functions. Math. Comp., 70(235):1183-1194 (electronic), 2001.

[44] A. Gil, J. Segura, and N. M. Temme. Algorithm 819: AIZ, BIZ: two Fortran 77 routines for the computation of complex Airy functions. ACM Trans. Math. Software, 28(3):325-336, 2002.

[45] A. Gil, J. Segura, and N. M. Temme. Computing complex Airy functions by numerical quadrature. Numer. Algorithms, 30(1):11-23, 2002.

[46] A. Gil, J. Segura, and N. M. Temme. Algorithm 831: Modified Bessel functions of imaginary order and positive argument. ACM Trans. Math. Software, 30(2):159-164, 2004.

[47] A. Gil, J. Segura, and N. M. Temme. Computing solutions of the modified Bessel differential equation for imaginary orders and positive arguments. ACM Trans. Math. Software, 30(2):145-158, 2004.

[48] A. Gil, J. Segura, and N. M. Temme. The ABC of hyper recursions. J. Comput. Appl. Math., 190(1-2):270-286, 2006.

[49] A. Gil, J. Segura, and N. M. Temme. Fast and accurate computation of the Weber parabolic cylinder function $w(a, x)$. 2009. Submitted to J. Comput. Phys.

[50] A. Gil, J. Segura, and N.M. Temme. Algorithm 850: Real parabolic cylinder functions $U(a, x), V(a, x)$. ACM Transactions on Mathematical Software, 32(1):102-112, March 2006.

[51] A. Gil, J. Segura, and N.M. Temme. Computing the real parabolic cylinder functions $U(a, x), V(a, x)$. ACM Transactions on Mathematical Software, 32(1):70-101, March 2006.

[52] A. Gil, J. Segura, and N.M. Temme. Numerically satisfactory solutions of hypergeometric recursions. Math. Comp., 76(259):1449-1468, 2007.

[53] Amparo Gil, Javier Segura, and Nico M. Temme. Computing toroidal functions for wide ranges of the parameters. J. Comput. Phys., 161(1):204-217, 2000.

[54] Amparo Gil, Javier Segura, and Nico M. Temme. Algorithm 822: GIZ, HIZ: two Fortran 77 routines for the computation of complex Scorer functions. ACM Trans. Math. Software, 28(4):436-447, 2002.

[55] Amparo Gil, Javier Segura, and Nico M. Temme. Numerical methods for special functions. Society for Industrial and Applied Mathematics (SIAM), Philadelphia, PA, 2007. 
[56] Amparo Gil, Javier Segura, and Nico M. Temme. Computing the conical function $P_{-1 / 2+i \tau}^{\mu}(x)$. SIAM J. Sci. Comput., 31(3):1716-1741, 2009.

[57] G. H. Golub and J. H. Welsch. Calculation of Gauss quadrature rules. Math. Comp., 23(106):221-230, 1969. Loose microfiche suppl. A1-A10.

[58] J. Grad and E. Zakrajšek. Method for evaluation of zeros of Bessel functions. J. Inst. Math. Appl., 11:57-72, 1973.

[59] S. Graffi and V. Grecchi. Borel summability and indeterminacy of the Stieltjes moment problem: application to the anharmonic oscillators. J. Mathematical Phys., 19(5):1002-1006, 1978.

[60] P. R. Graves-Morris, D. E. Roberts, and A. Salam. The epsilon algorithm and related topics. J. Comput. Appl. Math., 122(1-2):51-80, 2000. Numerical analysis 2000, Vol. II: Interpolation and extrapolation.

[61] J. F. Hart, E. W. Cheney, C. L. Lawson, H. J. Maehly, C. K. Mesztenyi, J. R. Rice, H. C. Thacher, Jr., and C. Witzgall. Computer Approximations. SIAM Ser. in Appl. Math. John Wiley and Sons Inc., New York, 1968.

[62] H. H. H. Homeier. Scalar Levin-type sequence transformations. J. Comput. Appl. Math., 122(1-2):81-147, 2000. Numerical analysis 2000, Vol. II: Interpolation and extrapolation.

[63] Tobias Huber and Daniel Maître. HypExp 2, Expanding hypergeometric functions about half-integer parameters. Comput. Phys. Comm., 178(10):755$776,2008$.

[64] Y. Ikebe. The zeros of regular Coulomb wave functions and of their derivatives. Math. Comp., 29:878-887, 1975.

[65] T. Inghoff, S. Fritzsche, and B. Fricke. Maple procedures for the coupling of angular momenta. IV: Spherical harmonics. Comput. Phys. Commun., 139(3):297-313, 2001.

[66] J. H. Johnson and J. M. Blair. REMES2 - a Fortran program to calculate rational minimax approximations to a given function. Technical Report AECL-4210, Atomic Energy of Canada Limited. Chalk River Nuclear Laboratories, Chalk River, Ontario, 1973.

[67] Masao Kodama. Algorithm 877: A subroutine package for cylindrical functions of complex order and nonnegative argument. ACM Trans. Math. Software, 34(4):Art. 22, 21, 2008.

[68] D. Levin. Development of non-linear transformations of improving convergence of sequences. Internat. J. Comput. Math., 3:371-388, 1973.

[69] Jean Marie Linhart. Algorithm 885: Computing the logarithm of the normal distribution. ACM Trans. Math. Software, 35(3):Art. 20, 2008. 
[70] D. W. Lozier and F. W. J. Olver. Numerical evaluation of special functions. In Mathematics of Computation 1943-1993: a half-century of computational mathematics (Vancouver, BC, 1993), volume 48 of Proc. Sympos. Appl. Math., pages 79-125. Amer. Math. Soc., Providence, RI, 1994. Updates are available at http://math.nist.gov/mcsd/Reports/2001/nesf/.

[71] Dan W. Lozier and F. W. J. Olver. Airy and Bessel functions by parallel integration of ODEs. In R. F. Sincovec, D. E. Keyes, Michael R. Leuze, Linda R. Petzold, and Daniel A. Reed, editors, Parallel processing for scientific computing, volume II of Proceedings of the Sixth SIAM Conference, pages 530-538, Philadelphia, PA, 1993. Society for Industrial and Applied Mathematics (SIAM).

[72] Y. L. Luke. The special functions and their approximations II. Vol. 53 of Mathematics in Science and Engineering. Academic Press, New York, 1969.

[73] Y. L. Luke. Mathematical functions and their approximations. Academic Press, New York, 1975.

[74] A. J. MacLeod. An instability problem in Chebyshev expansions for special functions. ACM SigNum Newsletter, 28(2):2-7, 1993.

[75] G. Maino, E. Menapace, and A. Ventura. Computation of parabolic cylinder functions by means of a Tricomi expansion. J. Comput. Phys., 40(2):294-304, 1981.

[76] G. Meinardus. Approximation of functions: theory and numerical methods, volume 13 of Springer Tracts in Natural Philosophy. Springer-Verlag, New York, 1967. Expanded translation from the German edition. Translated by Larry L. Schumaker.

[77] N. Michel. Precise Coulomb wave functions for a wide range of complex $l, \eta$ and z. Comput. Phys. Commun., 176:232-249, 2007.

[78] N. Michel and M.V. Stoitsov. Fast computation of the Gauss hypergeometric function with all its parameters complex with application to the Pöschl-Teller-Ginocchio potential wave functions. Comput. Phys. Commun., 178:535-551, 2008.

[79] Stephen Lloyd Baluk Moshier. Methods and programs for mathematical functions. Ellis Horwood Series: Mathematics and its Applications. Ellis Horwood Ltd., Chichester, 1989.

[80] A. Murli and M. Rizzardi. Algorithm 682: Talbot's method for the Laplace inversion problem. ACM Trans. Math. Software, 16(2):158-168, 1990.

[81] C. J. Noble. Evaluation of negative energy Coulomb (Whittaker) functions. Comput. Phys. Comm., 159(1):55-62, 2004.

[82] A. B. Olde Daalhuis and F. W. J. Olver. On the asymptotic and numerical solution of linear ordinary differential equations. SIAM Rev., 40(3):463-495, 1998. 
[83] R. B. Paris and A. D. Wood. Stokes phenomenon demystified. Bull. Inst. Math. Appl., 31(1-2):21-28, 1995.

[84] M. J. D. Powell. On the maximum errors of polynomial approximations defined by interpolation and by least squares criteria. Comput. J., 9(4):404407, 1967.

[85] William H. Press, Saul A. Teukolsky, William T. Vetterling, and Brian P. Flannery. Numerical recipes in $\mathrm{C}++$. Cambridge University Press, Cambridge, 2002. The art of scientific computing, Second edition, updated for $\mathrm{C}++$.

[86] J. R. Rice. The approximation of functions. Vol. I: Linear theory. AddisonWesley Publishing Co., Reading, MA-London, 1964.

[87] M. Rizzardi. A modification of Talbot's method for the simultaneous approximation of several values of the inverse Laplace transform. ACM Trans. Math. Software, 21(4):347-371, 1995.

[88] Thomas Schmelzer and Lloyd N. Trefethen. Computing the gamma function using contour integrals and rational approximations. SIAM J. Numer. Anal., 45(2):558-571 (electronic), 2007.

[89] J. L. Schonfelder. Chebyshev expansions for the error and related functions. Math. Comp., 32(144):1232-1240, 1978.

[90] Z. Schulten, D. G. M. Anderson, and Roy G. Gordon. An algorithm for the evaluation of the complex Airy functions. J. Comput. Phys., 31(1):60-75, 1979.

[91] Z. Schulten, R. G. Gordon, and D. G. M. Anderson. A numerical algorithm for the evaluation of Weber parabolic cylinder functions $U(a, x), V(a, x)$, and $W(a, \pm x)$. J. Comput. Phys., 42(2):213-237, 1981.

[92] M. J. Seaton. Coulomb functions for attractive and repulsive potentials and for positive and negative energies. Comput. Phys. Comm., 146(2):225-249, 2002.

[93] M. J. Seaton. FGH, a code for the calculation of Coulomb radial wave functions from series expansions. Comput. Phys. Comm., 146(2):250-253, 2002.

[94] M. J. Seaton. NUMER, a code for Numerov integrations of Coulomb functions. Comput. Phys. Comm., 146(2):254-260, 2002.

[95] J. Segura. Reliable computation of the zeros of solutions of second order linear ODEs with a fourth order method. Submitted to SIAM J. Numer. Anal., Jan. 2009.

[96] J. Segura. The zeros of special functions from a fixed point method. SIAM J. Numer. Anal., 40(1):114-133 (electronic), 2002. 
[97] J. Segura, P. Fernández de Córdoba, and Yu. L. Ratis. A code to evaluate modified Bessel functions based on the continued fraction method. Comput. Phys. Comm., 105(2-3):263-272, 1997.

[98] J. Segura and A. Gil. Parabolic cylinder functions of integer and half-integer orders for nonnegative arguments. Comput. Phys. Comm., 115(1):69-86, 1998.

[99] Javier Segura and Nico M. Temme. Numerically satisfactory solutions of Kummer recurrence relations. Numer. Math., 111(1):109-119, 2008.

[100] David M. Smith. Algorithm 814: Fortran 90 software for floating-point multiple precision arithmetic, gamma and related functions. ACM Trans. Math. Softw., 27(4):377-387, 2001.

[101] A. Talbot. The accurate numerical inversion of Laplace transforms. J. Inst. Math. Appl., 23(1):97-120, 1979.

[102] J. D. Talman. NumSBT: A subroutine for calculating spherical Bessel transforms numerically. Comput. Phys. Comm., 180(2):332-338, 2009.

[103] N. M. Temme. On the numerical evaluation of the modified Bessel function of the third kind. J. Computational Phys., 19(3):324-337, 1975.

[104] N. M. Temme. An algorithm with ALGOL 60 program for the computation of the zeros of ordinary Bessel functions and those of their derivatives. $J$. Comput. Phys., 32:270-279, 1979.

[105] N. M. Temme. Special functions. Wiley-Interscience, New York, 1996. An introduction to the classical functions of mathematical physics.

[106] N. M. Temme. Numerical algorithms for uniform Airy-type asymptotic expansions. Numer. Algorithms, 15(2):207-225, 1997.

[107] W.J. Thompson. An Atlas for Computing Mathematical Functions: An Illustrated Guide for Practitioners, with program in Fortran 90 and Mathematica. Wiley-Interscience, New York, 1997.

[108] Loyd N. Trefethen, J.A.C. Weideman, and Thomas Schmelzer. Talbot quadrature and rational approximations. Technical report, Oxford University Computing Laboratory Numerical Analysis Group, 2005.

[109] Joris Van Deun and Ronald Cools. Algorithm 858: computing infinite range integrals of an arbitrary product of Bessel functions. ACM Trans. Math. Software, 32(4):580-596, 2006.

[110] C. Van Loan. Computational frameworks for the fast Fourier transform, volume 10 of Frontiers in Applied Mathematics. Society for Industrial and Applied Mathematics (SIAM), Philadelphia, PA, 1992.

[111] Z. X. Wang and D. R. Guo. Special functions. World Scientific, Teaneck, NJ, 1989. Translated from the Chinese by Guo and X. J. Xia. 
[112] J. A. C. Weideman and L. N. Trefethen. Parabolic and hyperbolic contours for computing the Bromwich integral. Math. Comp., 76(259):1341-1356 (electronic), 2007.

[113] J.A.C. Weideman. Optimizing Talbot's contours for the inversion of the Laplace transform. Technical Report NA 05/05, Oxford U. Computing Lab., 2005.

[114] E. J. Weniger. Nonlinear sequence transformations for the acceleration of convergence and the summation of divergent series. Computer Physics Reports, 10(5,6):189-371, 1989.

[115] E. J. Weniger. On the summation of some divergent hypergeometric series and related perturbation expansions. J. Comput. Appl. Math., 32(1-2):291300, 1990. Extrapolation and rational approximation (Luminy, 1989).

[116] E. J. Weniger and J. Č́žzek. Rational approximations for the modified Bessel function of the second kind. Comput. Phys. Comm., 59(3):471-493, 1990.

[117] E. J. Weniger, J. Č́žzek, and F. Vinette. The summation of the ordinary and renormalized perturbation series for the ground state energy of the quartic, sextic, and octic anharmonic oscillators using nonlinear sequence transformations. J. Math. Phys., 34(2):571-609, 1993.

[118] E.J. Weniger. Computation of the Whittaker function of the second kind by summing its divergent asymptotic series with the help of nonlinear sequence transformations. Comput. Phys., 10:496-503, 1996.

[119] L. Wuytack. Commented bibliography on techniques for computing Padé approximants. In Padé approximation and its applications (Proc. Conf., Univ. Antwerp, Antwerp, 1979), volume 765 of Lecture Notes in Math., pages 375392. Springer, Berlin, 1979.

[120] P. Wynn. On a device for computing the $e_{m}\left(S_{n}\right)$ tranformation. Math. Tables Aids Comput., 10:91-96, 1956.

[121] P. Wynn. Upon systems of recursions which obtain among the quotients of the Padé table. Numer. Math., 8(3):264-269, 1966.

[122] Shanjie Zhang and Jianming Jin. Computation of special functions. WileyInterscience Publication, New York, 1996. 